\title{
Modelling the Impact of Delaying Vaccination Against SARS-CoV-2 Assuming Unlimited Vaccines Supply
}

Eduardo Massad ( $\nabla$ edmassad@dim.fm.usp.br)

Universidade de Sao Paulo https://orcid.org/0000-0002-7200-2916

Marcos Amaku

University of Sao Paulo: Universidade de Sao Paulo

Dimas Tadeu Covas

Instituto Butantan: Instituto Butantan

Francisco Antonio Bezerra Coutinho

Universidade de Sao Paulo Campus de Sao Paulo: Universidade de Sao Paulo

Raymundo Soares Azevedo

Universidade de Sao Paulo Campus de Sao Paulo: Universidade de Sao Paulo

Research

Keywords: COVID-19, SARS-CoV-2, Mathematical Models, Vaccines

Posted Date: June 15th, 2021

DOl: https://doi.org/10.21203/rs.3.rs-605245/v1

License: (9) This work is licensed under a Creative Commons Attribution 4.0 International License.

Read Full License 
Modelling the Impact of Delaying VaccinationAgainst SARS-CoV-2 Assuming Unlimited Vaccines Supply

Marcos Amaku ${ }^{1,4}$, Dimas Tadeu Covas ${ }^{2}$, Francisco Antonio Bezerra Coutinho ${ }^{1}$, Raymundo Soares Azevedo $^{1}$, Eduardo Massad ${ }^{1,3,5, *}$.

${ }^{1}$ School of Medicine, University of Sao Paulo and LIM01-HCFMUSP, Sao Paulo, Brazil;

${ }^{2}$ Instituto Butantan, Sao Paulo, Brazil;

${ }^{3}$ School of Applied Mathematics, FundacaoGetulio Vargas, Rio de Janeiro, Brazil;

${ }^{4}$ School of Veterinary Medicine, University of Sao Paulo, Sao Paulo, Brazil;

${ }^{5}$ London School of Hygiene and TropicalMedicine, UK

*CorrespondingAuthor: SchoolofAppliedMathematics, FundacaoGetulio Vargas, Rua Praia de Botafogo 190, Rio de Janeiro, CEP 22250-900, RJ, Brazil, eduardo.massad@fgv.br 


\begin{abstract}
Background

At the moment we have more than 109 million cases and 2.4 million deaths around the world and vaccination represents the only hope to control the pandemic. Imperfections in planning vaccine acquisition and difficulties in implementing distribution among the population, however, have hampered the control of the virus so far.
\end{abstract}

\title{
Methods
}

We propose a new mathematical model to estimate the impact of vaccination delay against the 2019 coronavirus induces disease (COVID-19) on the number of cases and deaths by the disease in Brazil. We apply the model to Brazil as a whole and to the State of Sao Paulo, the most affected by COVID19 in Brazil. We simulated the model for the populations of the State of Sao Paulo and Brazil as a whole, varying the scenarios related to vaccine efficacy and compliance from the populations.

\section{Results}

The model projects that, in the absence of vaccination, almost 170 thousand deaths and more than 350 thousand deaths until the end of 2021 for Sao Paulo and Brazil, respectively. If in contrast, Sao Paulo and Brazil had enough vaccine supply and so started a vaccination campaign in January with the maximum vaccination rate, compliance and efficacy, they could have averted more than 112 thousand deaths and 127 thousand deaths, respectively. In addition, that for each month of delay the number of deaths increases monotonically in a logarithm fashion, for both the State of Sao Paulo and Brazil as a whole.

\section{Conclusions}

Our model shows that the current delay in the vaccination schedules that is observed in many countries has serious consequences in terms of mortality by the disease and should serve as an alert to health authorities to speed the process up such that the highest number of people to be immunized is reached in the shortest period of time.

Keywords: COVID-19; SARS-CoV-2; Mathematical Models; Vaccines. 


\section{Introduction}

As the world struggles to implement vaccination schemes against the Severe acute respiratory syndrome by the coronavirus 2 (SARS-CoV-2), limited production of doses, imperfections in planning vaccine acquisition and difficulties in implementing distribution among the population, have hampered the control of the virus so far [1]. As of 18 February 2021, 188 million people have been vaccinated around the world, which represents less than $3 \%$ of the total. In Brazil, the total number of vaccinated people so far is, around $2.5 \%$ of the target population [2]. The world vaccination rate currently is less than 4 million doses per day, a very small rate [3]. So, it is no surprise that vaccination by itself has had so far little effect on the number of cases and deaths that continues to soar in many countries. At the moment we have more than 109 million cases and 2.4 million deaths around the world [4].

Although previous pandemics have demonstrated that pharmaceutical interventions are less important than non-pharmaceutical intervention in controlling the infection, there is a growing body of evidences that this will not be the case with the vaccines against COVID-19 [5-8].

Immediately after the emergence of SARS-CoV-2 in China, many laboratories around the world started the development of more than 100 types of different vaccines, reducing in less than one year the usual time frame of new vaccines development and testing, which normally would be around ten years, a remarkable effort [6], [9].

There is a wide range of covid-19 vaccines being developed [10]. As of February 2021, there are 80 vaccine candidates in 212 clinical trials and 11 vaccines approved by at least one country [10].Of these, 80 vaccines are in the pipeline, of which 20 are in Phase 3 of clinical trials (four have already completed this phase) and 37in Phase 2 [10]. In the United States of America (USA), three vaccines completed Phase 3 trials, namely, Moderna, Pfizer, and Oxford-AstraZeneca, and two are still in Phase 3 [10].

However, in order to have significant impact on the course of the pandemic, safe and effective vaccines have to emerge in less time that it would take the affected populations to reach natural herd immunity because to wait to have herd natural immunity would result in millions of deaths. Therefore, an unprecedented time-schedule to roll out any effective vaccine is urgently needed. Nevertheless, in many countries the vaccination is limited to certain individual groups and the 
distribution of enough doses for these individuals is very slow. We have at the moment only 188 million doses applied in 64 countries around the world [11].

Mathematical models have played a key role in helping understanding of COVID-19 dynamics as well as in determining the best decisions about mitigation strategies [12]. In this sense, models remain essential tools for evidence synthesis, planning and forecasting and decision analysis for COVID-9 control and policymaking [12].

In the context of vaccination, the limited initial supply of COVID-19 vaccines raises the question on low to prioritize doses [13]. In addition, there is a conflict between infection and vaccination with infections still growing exponentially in many countries around the world, whereas vaccination rates are inherently restricted by supply and logistics [14]. It is, therefore, a need of a model-informed approach to quantify the impact of COVID-19 vaccination on the course of the epidemic [13].

Brazil has accumulated almost 10 million cases and more than 240 thousand deaths at the time of writing (18 February 2021) [15]. The state of Sao Paulo, the most populous in Brazil reported almost 2 million cases and 57 thousand deaths so far [16]. Notwithstanding the fact that Brazil is the third country with the highest number of cases and second with the highest number of deaths in the world, only two vaccines, Coronavac and Oxford-AstraZeneca have been licensed. Currently, just above $2 \%$ of the target population have received a first dose of one or the other vaccines [17].

This paper proposes a new model to estimate the impact of vaccination delay against Covid-19 on the number of cases and deaths by the disease in Brazil. We apply the model to Brazil as a whole and to the State of Sao Paulo, the most affected by COVID-19 in Brazil. This work is a theoretical exercise because it assumes that at every time of the pandemic there is enough vaccine, which is not realistic for the majority of countries.

\section{The model}

The model is an extension of the one presented in [18] and has the following variables:

1) Susceptible individuals, denoted $S(t)$, which can either be vaccinated with rate $v$, or acquire the infection with rate $\beta$. Susceptible are born with rate $\Lambda$ and die by other causes with rate $\mu$;

2) Vaccinated individuals, denoted $V(t)$, which are transferred from the susceptible state with the vaccination rate $v$. The vaccine is assumed to have efficacy $q$ and a fraction $w$ of the susceptible complies with the vaccination policy. Vaccinated individuals die by other causes with rate $\mu$; 
3) Failure to be immunized individuals, denoted

. A fraction $(1-q)$ of those vaccinated susceptible fail to be immunized (that is, not completely immunized after vaccination), and can either acquire the infection with the same rate $\beta$ as those non-vaccinated susceptible or die by other causes with rate $\mu$;

4) Exposed individuals, denoted $E(t)$, are those individuals who acquired the infection but are still in the stage that precedes either the overtly diseased patients or the asymptomatic individuals (see below). Exposed individuals can either progress to an asymptomatic stage with rate $\delta_{\mathrm{A}}$, or to full-blown COVID-19 patients with rate $\delta_{\mathrm{I}}$ or die by other causes with rate $\mu$. A fraction $p_{\mathrm{E}}$ of those exposed are infective to susceptibles;

5) Asymptomatic (or pauci-symptomatic) individuals, denoted $A(t)$, who progressed from the exposed and are, therefore, infected with SARS-CoV-2 but show no or very few symptoms. Asymptomatic individuals can either die by natural causes or by the infection, with rates $\mu$ and $\alpha_{\mathrm{A}}$, respectively, or recover from the infection with rate $\gamma_{\mathrm{A}}$. A fraction $p_{\mathrm{A}}$ of these asymptomatic individuals are infective to susceptible;

6) Infective individuals, denoted $I(t)$, are those individuals infected with SARS-CoV-2 and who show the characteristic clinical signs and symptoms of COVID-19. Infective individuals can either die by natural causes or by the infection, with rates $\mu$ and $\alpha_{I}$, respectively, or recover from the infection with rate $\gamma_{\mathrm{I}}$, or progress to hospitalized $(H(t))$ or critically ill stages $(G(t))$ (see below) with rates $\sigma_{\mathrm{H}}$ and $\sigma_{\mathrm{G}}$, respectively;

7) Hospitalized individuals, denoted $H(t)$, are individuals with full-blown COVID-19 but who do not require Intensive Care Unit support. These individuals candie by natural causes or by the infection, with rates $\mu$ and $\alpha_{\mathrm{H}}$, respectively, or recover from the infection with rate $\gamma_{\mathrm{H}}$;

8) Grave patients, denoted $G(t)$, are seriously ill patients requiring Intensive Care respiratory support. These individuals candie by natural causes or by the infection, with rates $\mu$ and $\alpha_{H}$, respectively, or recover from the infection with rate $\gamma_{G}$; and finally

9) Recovered individuals, denoted $R(t)$, are those individuals who recovered from the infection. They can die by natural causes with rate $\mu$.

Figure 1 shows a diagram with the model's stages and transitions. 


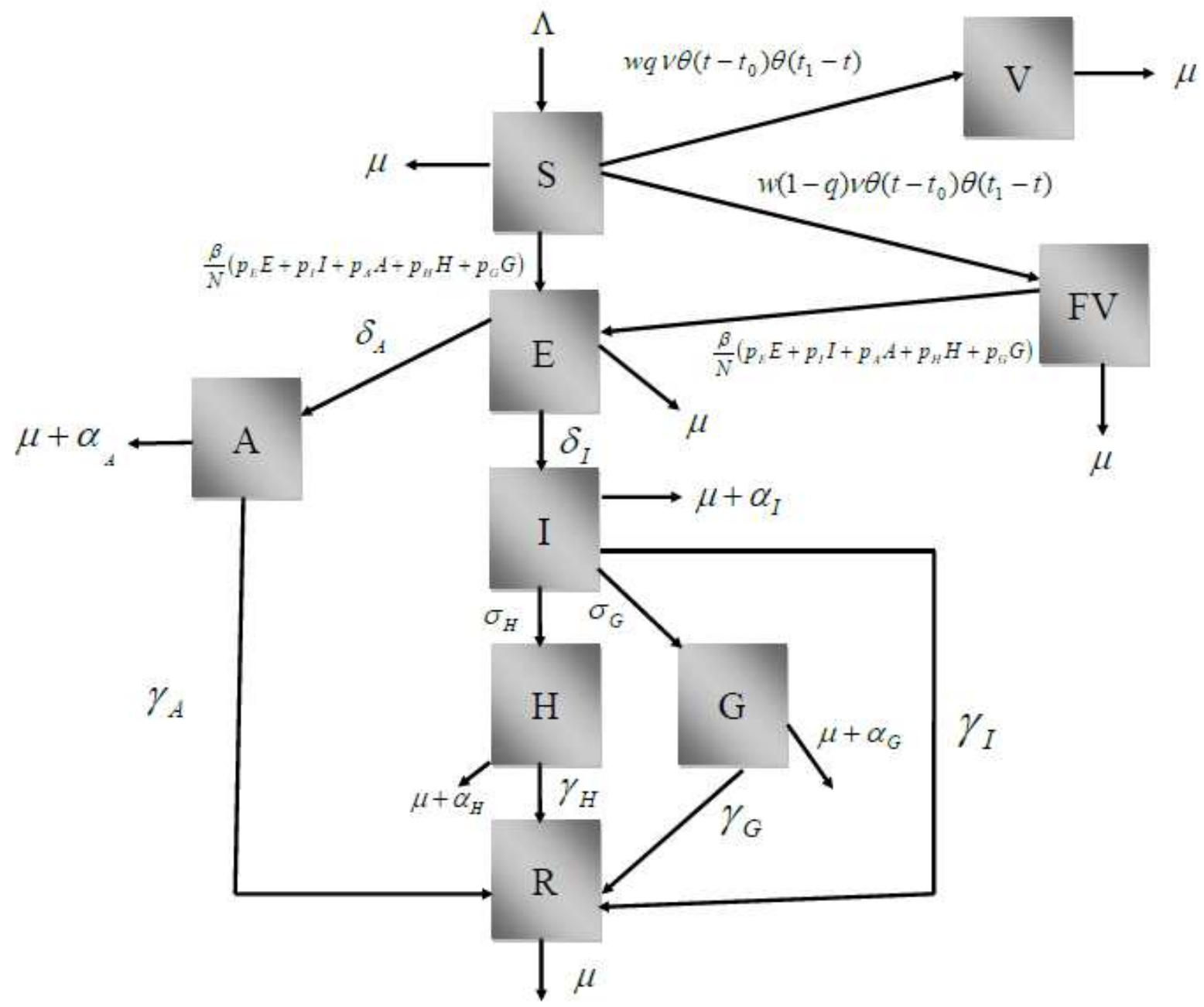

Figure 1. Diagram showing the model's stages and transitions. The figure shows a block diagram with all the model's states, namely, susceptible $(\mathrm{S})$, vaccinated $(\mathrm{V})$, vaccination failure $(\mathrm{FV})$, exposed to the virus $(E)$, asymptomatic $(A)$, infected and infectious $(I)$, hospitalized $(H)$, ICU patients $(G)$ and recovered from infection $(R)$. Transitions from one compartment to other are represented as rates, as described in the main text.

The model is described by the following set of equations:

$$
\begin{aligned}
& \begin{aligned}
\frac{d S(t)}{d t}=-\frac{\beta}{N} S(t)\left[p_{E} E(t)+p_{A} A(t)+p_{I} I(t)+p_{H} H(t)+p_{G} G(t)\right]-v w S(t) \theta\left(t-t_{0}\right) \theta\left(t_{1}-t\right) \\
\quad+\Lambda(\mathrm{t})-\mu S(t)
\end{aligned} \\
& \begin{aligned}
\frac{d E(t)}{d t}= & \frac{\beta}{N}[S(t)+F V(t)]\left[p_{E} E(t)+p_{A} A(t)+p_{I} I(t)+p_{H} H(t)+p_{G} G(t)\right]-\left(\delta_{A}+\delta_{I}+\mu\right) E(t)
\end{aligned} \\
& \begin{aligned}
\frac{d A(t)}{d t}= & \delta_{A} E(t)-\left(\gamma_{A}+\alpha_{A}+\mu\right) A(t)
\end{aligned} \\
& \frac{d I(t)}{d t}=\delta_{I} E(t)-\left(\sigma_{H}+\sigma_{G}+\gamma_{I}+\alpha_{I}+\mu\right) I(t)
\end{aligned}
$$




$$
\begin{aligned}
& \frac{d H(t)}{d t}=\sigma_{H} I(t)-\left(\gamma_{H}+\alpha_{H}+\mu\right) H(t) \mu \\
& \frac{d G(t)}{d t}=\sigma_{G} I(t)-\left(\gamma_{G}+\alpha_{G}+\mu\right) G(t) \\
& \frac{d R(t)}{d t}=\gamma_{A} A(t)+\gamma_{I} I(t)+\gamma_{H} H(t)+\gamma_{G} G(t)-\mu R(t) \\
& \frac{d V(t)}{d t}=[v q w S(t)-\mu V(t)] \theta\left(t-t_{0}\right) \theta\left(t_{1}-t\right) \\
& \frac{d F V(t)}{d t}=\left\{v(1-q) w S(t)-\frac{\beta}{N} F V(t)\left[p_{E} E(t)+p_{A} A(t)+p_{I} I(t)+p_{H} H(t)+p_{G} G(t)\right]\right. \\
& N(t)=S(t)+E(t)+A(t)+I(t)+H(t)+G(t)+R(t)+V(t)+F V(t) \\
& \Lambda(\mathrm{t})=\mu N(t)
\end{aligned}
$$

In the model, the $\theta$ function is the Heaviside step-function, included to simulate different times vaccination has started.

The incidence, $\operatorname{Inc}(t)$, is given by:

$$
\begin{aligned}
\operatorname{Inc}(t)=\beta S( & t) \\
& +F V(t) \theta\left(t-t_{0}\right) \theta\left(t_{1}-t\right)\left[p_{E} E(t)+p_{A} A(t)+p_{I} I(t)+p_{H} H(t)\right. \\
& \left.+p_{G} G(t)\right]
\end{aligned}
$$

The total number of cases, Cases, is given by:

$$
\begin{aligned}
\text { Cases }=\int_{0}^{\infty} \frac{\beta}{N}\left\{[ S ( t ) + F V ( t ) \theta ( t - t _ { 0 } ) \theta ( t _ { 1 } - t ) ] \left[p_{E} E(t)+p_{A} A(t)+p_{I} I(t)+p_{H} H(t)\right.\right. \\
\left.\left.+p_{G} G(t)\right]\right\} d t
\end{aligned}
$$

The total number of deaths, Deaths due to COVID-19,is given by:

$$
\text { Deaths }=\int_{0}^{\infty}\left[\alpha_{A} A(t)+\alpha_{I} I(t)+\alpha_{H} H(t)+\alpha_{G} G(t)\right] d t
$$


The total number of vaccinated individuals, Vaccinated, is given by:

Vaccinated $=\int_{0}^{\infty}\left[v w S(t) \theta\left(t-t_{0}\right) \theta\left(t_{1}-t\right)\right] d t$

In table 1 we show the parameters used for the simulation of model (1) for Brazil and the State of São Paulo.

Table 1 - Parameters used in the model for the State of São Paulo and Brazil.

\begin{tabular}{|c|c|c|c|}
\hline Parameter & Description & \multicolumn{2}{|c|}{ Value } \\
\hline & & São Paulo & Brazil \\
\hline$\beta(t)$ & Potentially infective contact rate & \multicolumn{2}{|c|}{ Fitted (changes over time) } \\
\hline$p_{E}$ & Infectivity of exposed individuals & $0.4 *$ & $0.4 *$ \\
\hline$p_{I}$ & Infectivity of symptomatic individuals & $1.0 *$ & $1.0 *$ \\
\hline$p_{A}$ & Infectivity of asymptomatic individuals & $1 / 3 *$ & $1 / 3^{*}$ \\
\hline$p_{H}$ & Infectivity of hospitalized individuals & $0.01 *$ & $0.01 *$ \\
\hline$p_{G}$ & Infectivity of ICU patients & $0.01 *$ & $0.01 *$ \\
\hline$\mu$ & Natural mortality rate (life expectancy of 70 years) & $3.91 \times 10^{-5}$ days $^{-1} *$ & $3.91 \times 10^{-5}$ days $^{-1} *$ \\
\hline$\delta_{I}$ & Rate of evolution from exposed to infected & $1 / 2$ day $^{-1} *$ & $1 / 2 \mathrm{day}^{-1} *$ \\
\hline$\delta_{A}$ & Rate of evolution from exposed to asymptomatic & 0.874 day $^{-1} * *$ & 0.366 day $^{-1} * *$ \\
\hline$\gamma_{I}$ & Rate of recovery from infected & $1 / 3$ day $^{-1} *$ & $1 / 3 \mathrm{day}^{-1} *$ \\
\hline$\gamma_{A}$ & Rate of recovery from asymptomatic & $1 / 14$ day $^{-1} *$ & $1 / 14$ day $^{-1} *$ \\
\hline$\gamma_{H}$ & Rate of recovery from hospitalized & $1 / 10$ day $^{-1} *$ & $1 / 10$ day $^{-1} *$ \\
\hline$\gamma_{G}$ & Rate of recovery from ICU & 0.05 day $^{-1} * *$ & 0.0556 day $^{-1} * *$ \\
\hline$\alpha_{I}$ & Disease-induced mortality rate for infected individuals & $5 \times 10^{-4}$ day $^{-1} *$ & $3 \times 10^{-4}$ day $^{-1} *$ \\
\hline$\alpha_{A}$ & $\begin{array}{c}\text { Disease-induced mortality rate for asymptomatic } \\
\text { individuals }\end{array}$ & $0 *$ & $0 *$ \\
\hline$\alpha_{H}$ & Disease-induced mortality rate for hospitalized individuals & $10^{-4} \mathrm{day}^{-1} * *$ & $5.56 \times 10^{-4} \mathrm{day}^{-1} * *$ \\
\hline$\alpha_{G}$ & Disease-induced mortality rate for ICU patients & Fitted (cha & over time) \\
\hline$\sigma_{H}$ & Hospitalization rate & 0.12 day $^{-1} * *$ & $0.0518 \mathrm{day}^{-1} * *$ \\
\hline$\sigma_{G}$ & ICU admission rate & Fitted (cha & over time) \\
\hline$v$ & Vaccination rate & Variable (from $5 \times 1$ & ays $^{-1}$ to $10^{-1}$ days $^{-1}$ ) \\
\hline$q$ & Vaccination efficacy & & \\
\hline$w$ & Adherence to the vaccination campaign & & \\
\hline
\end{tabular}




\begin{tabular}{|ccc|}
\hline$K(t)$ & Notification ratio & Fitted (changes over time) \\
\hline$\Lambda(t)$ & Birth rate & Changes over time \\
\hline
\end{tabular}

*assumed; **fitted

In figure 2 we show the time that it would take to reach herd immunity ( $70 \%$ of the population, i.e $R_{0}=3.3$ ) as a function of the vaccination rate, $v$. This is calculated, approximately (neglecting mortality) by solving the system of equations:

$\frac{d S}{d t}=-v w q S$

$\frac{d V}{d t}=v w q S$

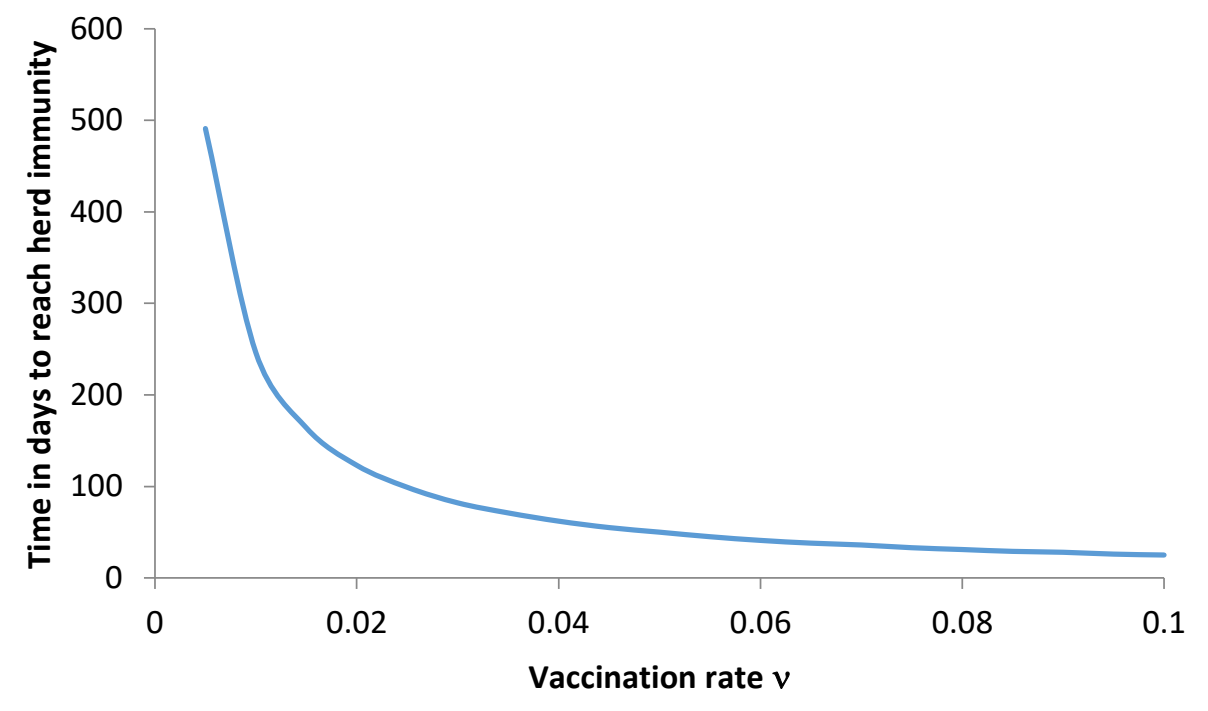

Figure 2 - Time in days taken to reach herd immunity as a function of vaccination rates as used in the simulation of the model.

\section{Results}

We simulated model (1) with the parameters as in table 1 for the two populations of the State of Sao Paulo and Brazil as a whole, varying the scenarios related to vaccine efficacy and compliance from the populations. We simulated efficacy with values of 50\%, 70\% and 90\% and compliance with values of $50 \%, 70 \%$ and $80 \%$. As mentioned above, we simulated the scenarios for Brazil as a whole and for the State of São Paulo. As shown in table 1, we have chosen values of vaccination rates that varied from 0.005 days $^{-1}$ until 0.1 days $^{-1}$. 
Below we show the results of the numerical simulations of the model.

We fitted the model parameters simultaneously to the data of cumulative number of reported cases and deaths(figure 3(a)) for Brazil and to the data of cumulative number of reported cases, deaths and the number of intensive care units (ICU) patients (figure 3(b)) for the State of Sao Paulo until December 18, 2020. The fitting procedure is described in [18-20].

(a) Brazil
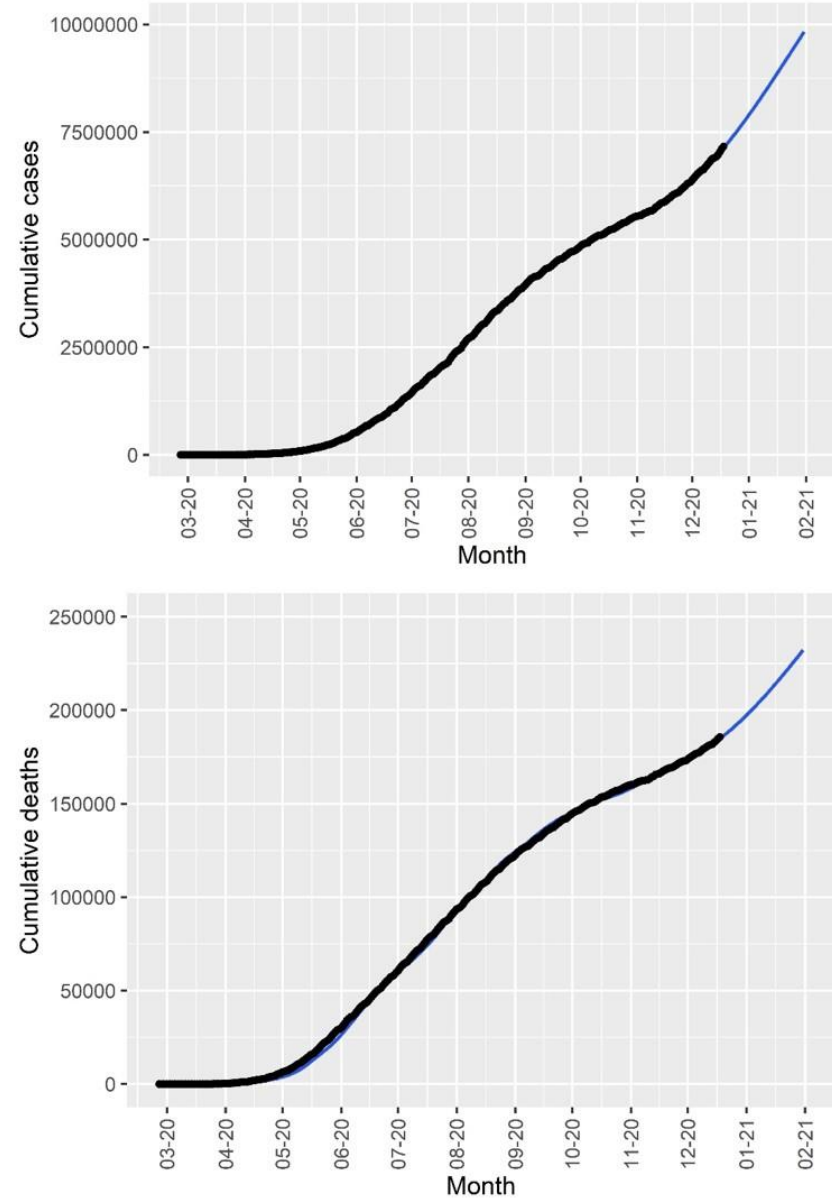

(b) Sao Paulo
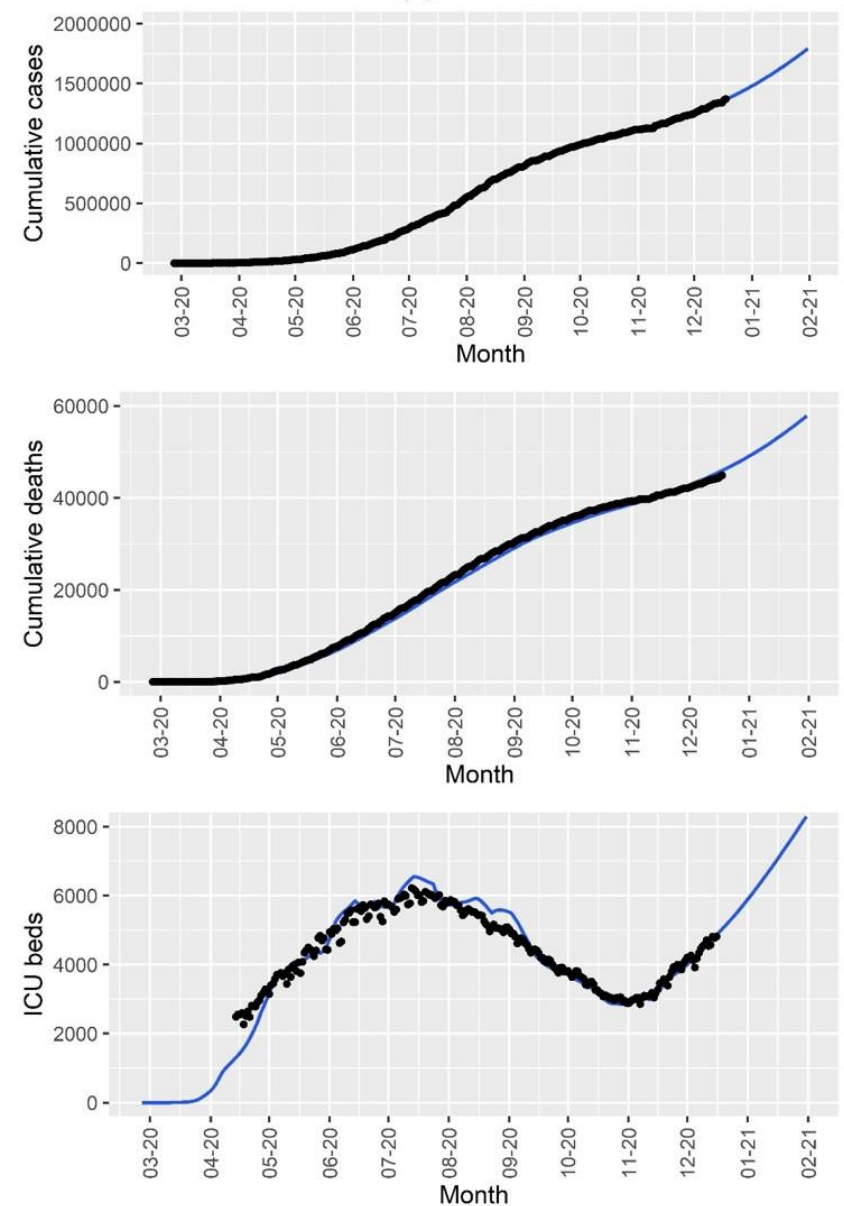

Figure 3 - Cumulative number of reported cases and deaths in (a) Brazil (black dots) and cases, deaths, and number of ICU patients in (b) the State of Sao Paulo. The blue lines correspond to the fitted models.

In figure 4 we show the percentage of averted deaths until $31^{\text {st }}$ December 2021 for several scenarios simulated and for mass vaccination starting on January $21^{\text {st }}$, February $21^{\text {st }}$, March $21^{\text {st }}$, April $21^{\text {st }}$ and May $21^{\text {st }}$, for 3 combinations population compliance to the campaign and vaccine efficacy. 
(a) Brazil

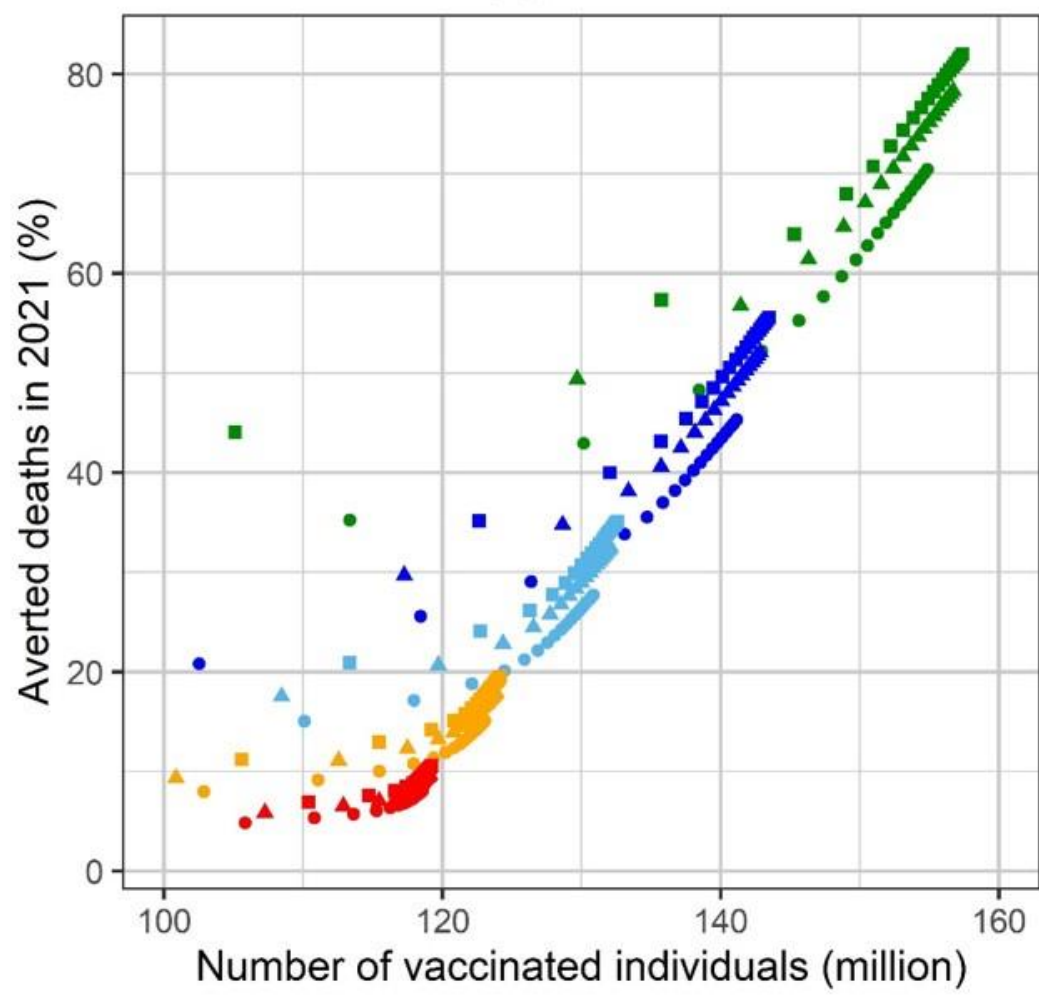

(b) Sao Paulo

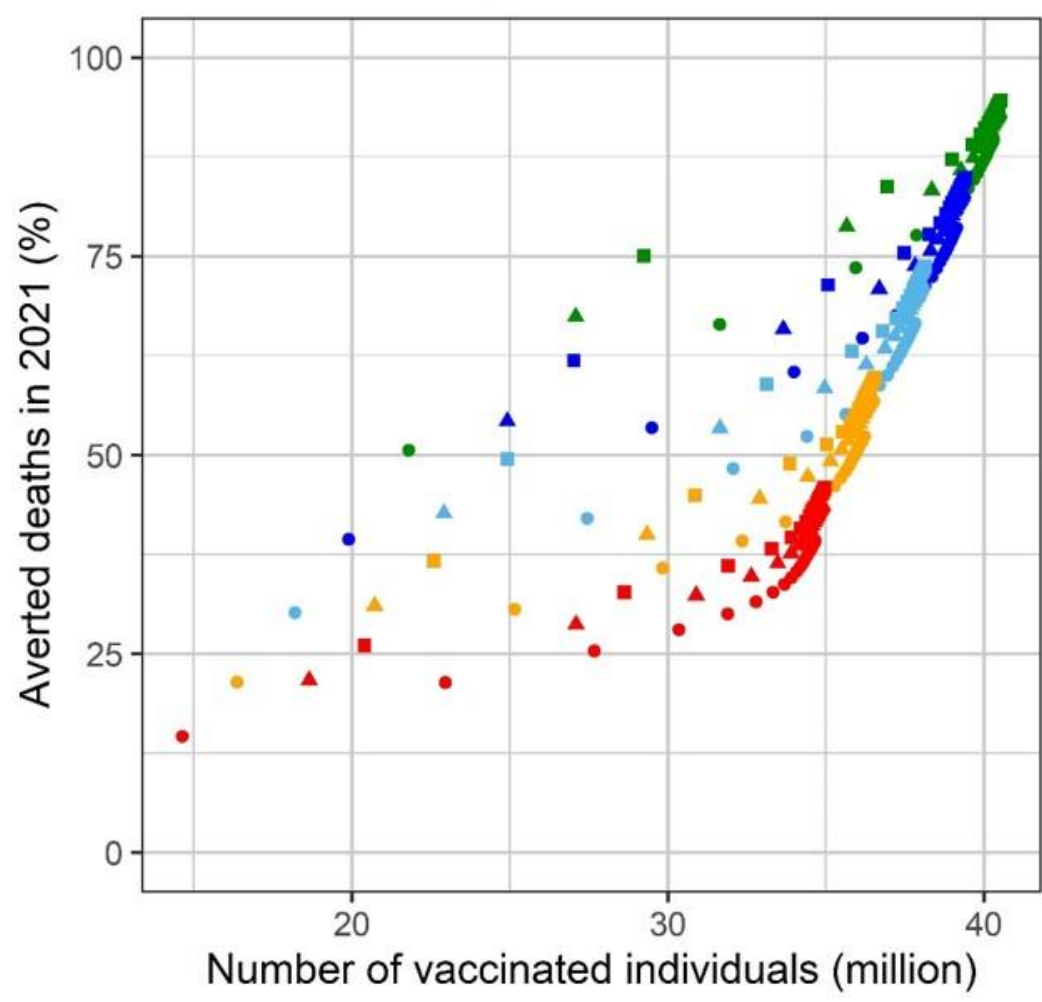

Start date

- Jan-21

- Feb-21

- Mar-21

- Apr-21

- May-21

Adherence ( $w$ ) efficacy (q)

- $w=0.5 q=0.5$

- $w=0.7 q=0.7$

- $w=0.8 q=0.9$

\section{Start date}

- Jan-21

- Feb-21

- Mar-21

- Apr-21

- May-21

Adherence ( $w$ ) efficacy (q)

- $w=0.5 q=0.5$

- $w=0.7 q=0.7$

- $w=0.8 q=0.9$

Figure 4 - Percentage of averted deaths in 2021 in (a) Brazil and (b) the State of Sao Paulo as a function of the number of vaccinated individuals for different start dates for the vaccination campaign. Three different combinations of vaccination adherence $(w)$ and vaccine efficacy $(q)$ were considered: 
$\mathrm{w}=0.8$ and $q=0.9$ (best-case scenario), $w=0.7$ and $q=0.7$ (baseline scenario) and $w=0.5$ and $q=0.5$ (worstcase scenario).

It can be noted from figure 4(a) that if Brazil had started a mass vaccination campaign on January $21^{\text {st }}$ with the maximum compliance of $80 \%$, a vaccine $90 \%$ efficacious, and a high vaccination rate, $80 \%$ of the expected deaths until December $31^{\text {st }}$ would be averted.

This result can also be seen in Figure 5 in which we show the percentage of averted deaths until the end of the year as a function of the vaccination rate for vaccination starting from January until May, with several vaccination scenarios, varying compliance, efficacy and date of the starting of the campaign. 
(a) Brazil

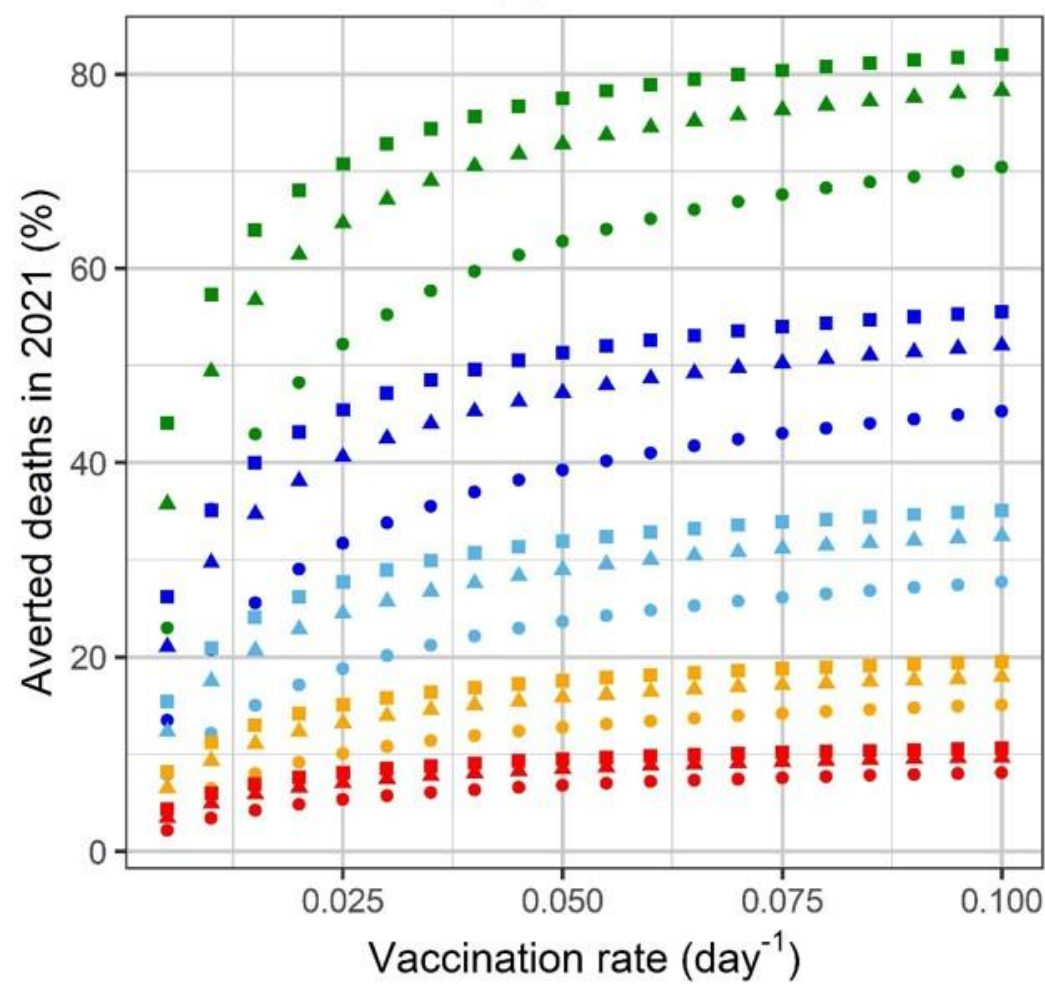

(b) Sao Paulo

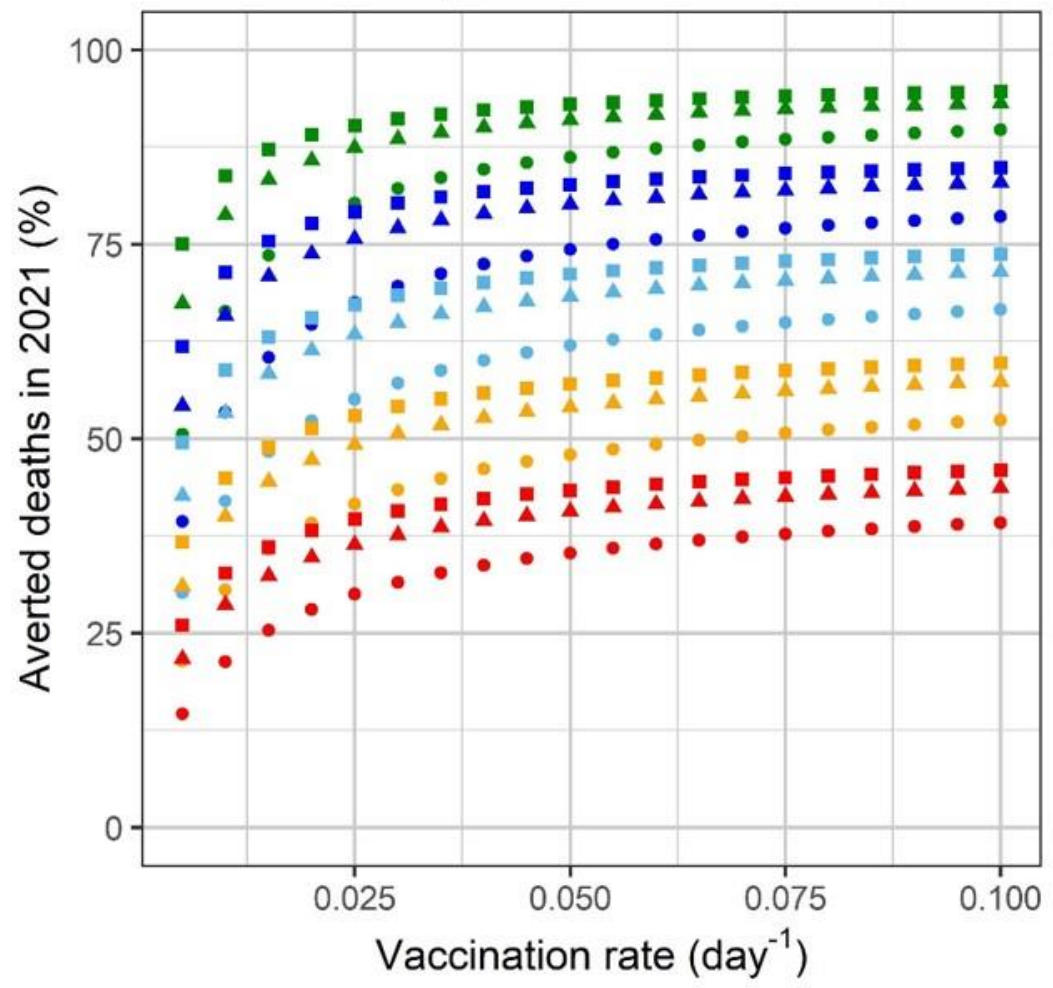

Start date

- Jan-21

- Feb-21

- Mar-21

- Apr-21

- May-21

Adherence (w) efficacy (q)

- $w=0.5 q=0.5$

- $w=0.7 q=0.7$

- $w=0.8 q=0.9$
Start date

- Jan-21

- Feb-21

- Mar-21

- Apr-21

- May-21

Adherence (w) efficacy (q)

- $w=0.5 q=0.5$

- $w=0.7 q=0.7$

- $\quad w=0.8 q=0.9$

Figure 5 - Percentage of averted deaths in 2021 in (a) Brazil and (b) the State of Sao Paulo as a function of the vaccination rate for different start dates for the vaccination campaign. Three different combinations of vaccination adherence $(w)$ and vaccine efficacy $(q)$ were considered: $w=0.8$ and $q=0.9$ (best-case scenario), $\mathrm{w}=0.7$ and $\mathrm{q}=0.7$ (baseline scenario) and $\mathrm{w}=0.5$ and $\mathrm{q}=0.5$ (worst-case scenario). 
In figure 6 we show the model's projection in terms of daily new cases and deaths for an intermediate vaccination rate and for the 5 different starting dates for the campaign. In the figure, we show the simulation with a vaccination rate of 0.05 days $^{-1}$. This implies in that with this rate the country would take approximately one year to reach the herd immunity, assumed to be $70 \%$ (i.e, a basic reproduction number equal to 3.3) of the population. In addition, this vaccination rate means 544 thousand vaccinations per day in Brazil, provided that there would be enough vaccine supply to this schedule.

(a) Brazil
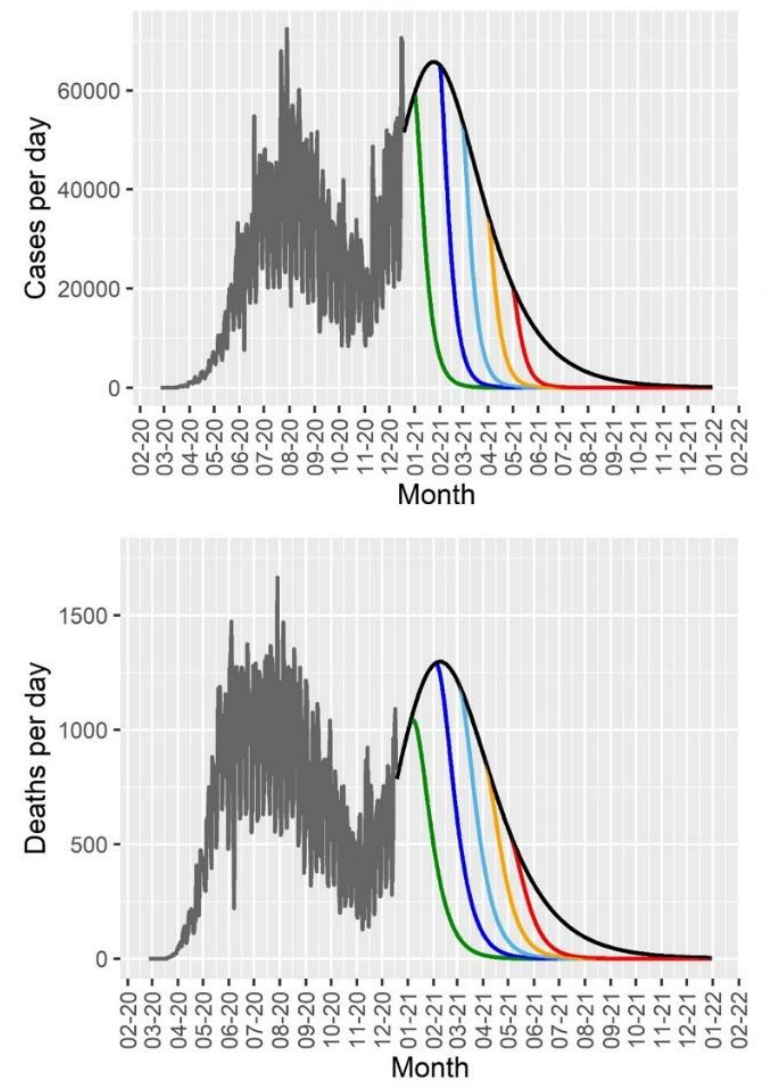

(b) Sao Paulo
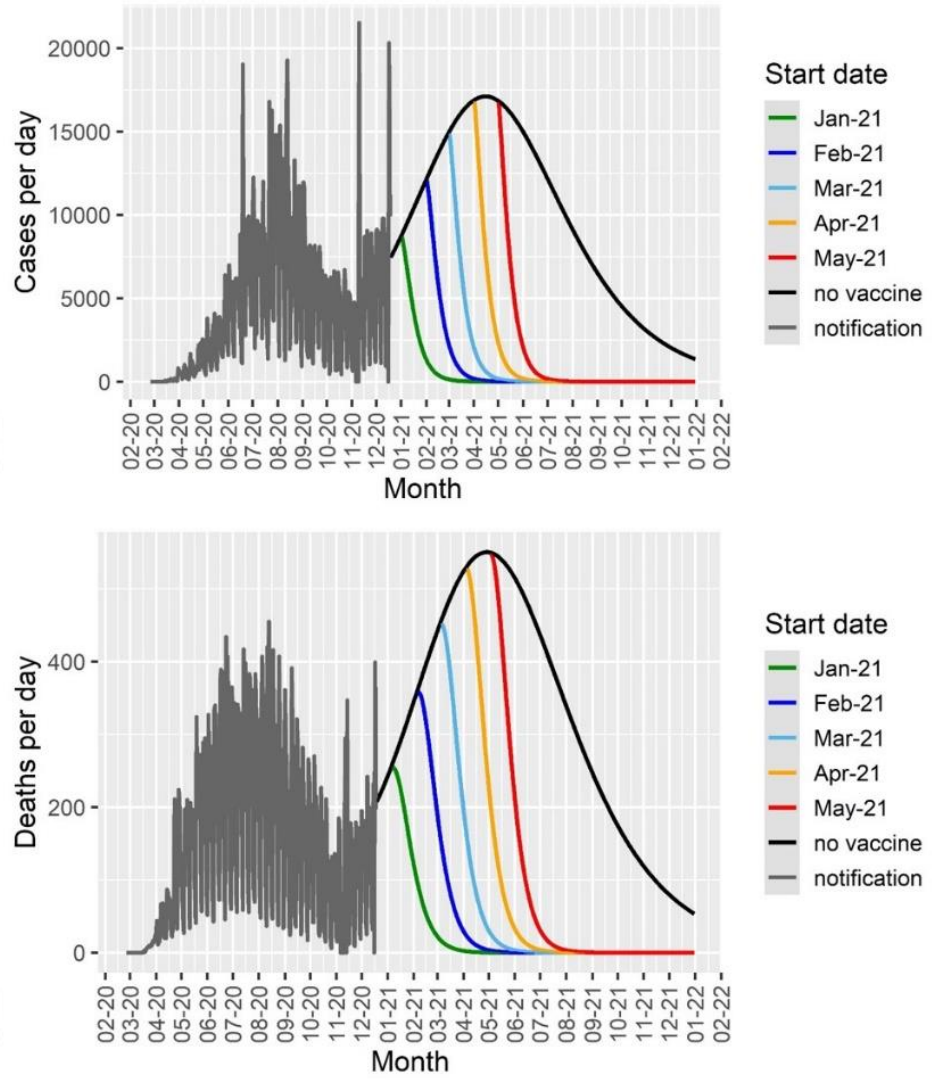

Figure 6 - Cases and deaths per day (b) as a function of time for different start dates for the vaccination campaign in (a) Brazil and (b) the State of Sao Paulo for a vaccination rate of $0.05 \mathrm{day}^{-1}$, and the results for the model with no vaccination (black lines). The notification data until December $18^{\text {th }} \mathbf{2 0 2 0}$ are shown in gray.

In figure 6 it is possible to observe the projected number of cases and deaths in the absence of vaccination and with the campaign beginning in January, February, March, April or May. 
In figure 7 we show the same simulated scenarios as in Figure 6 with a vaccination rate ten times lower.

(a) Brazil
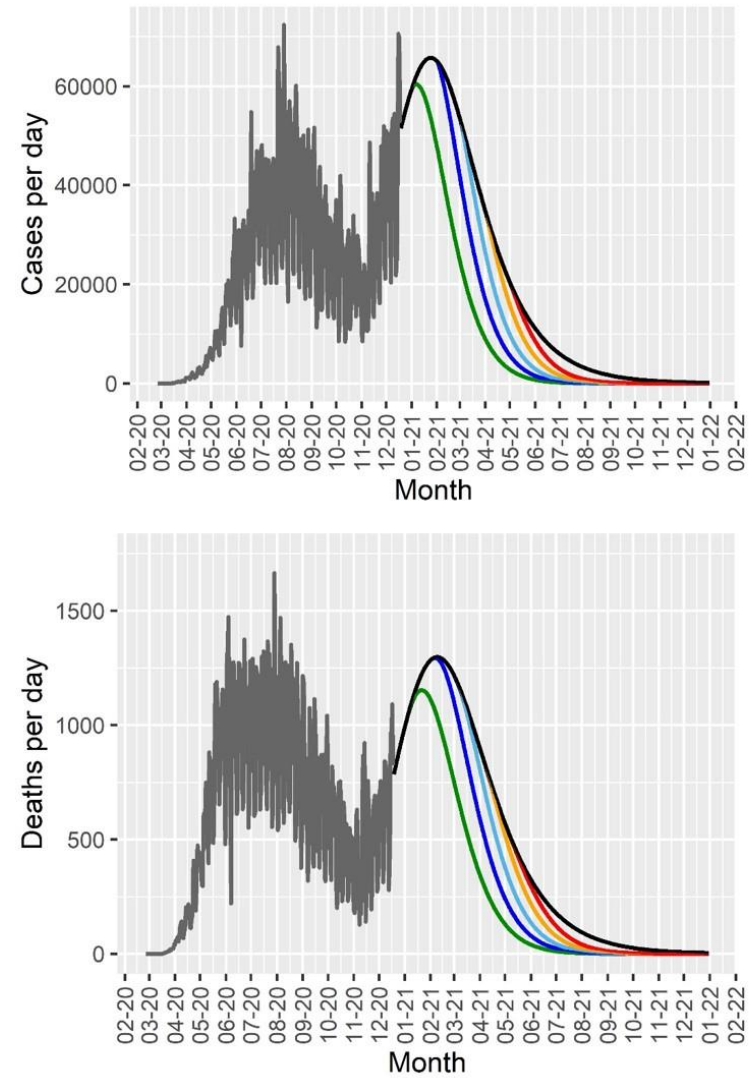

(b) Sao Paulo
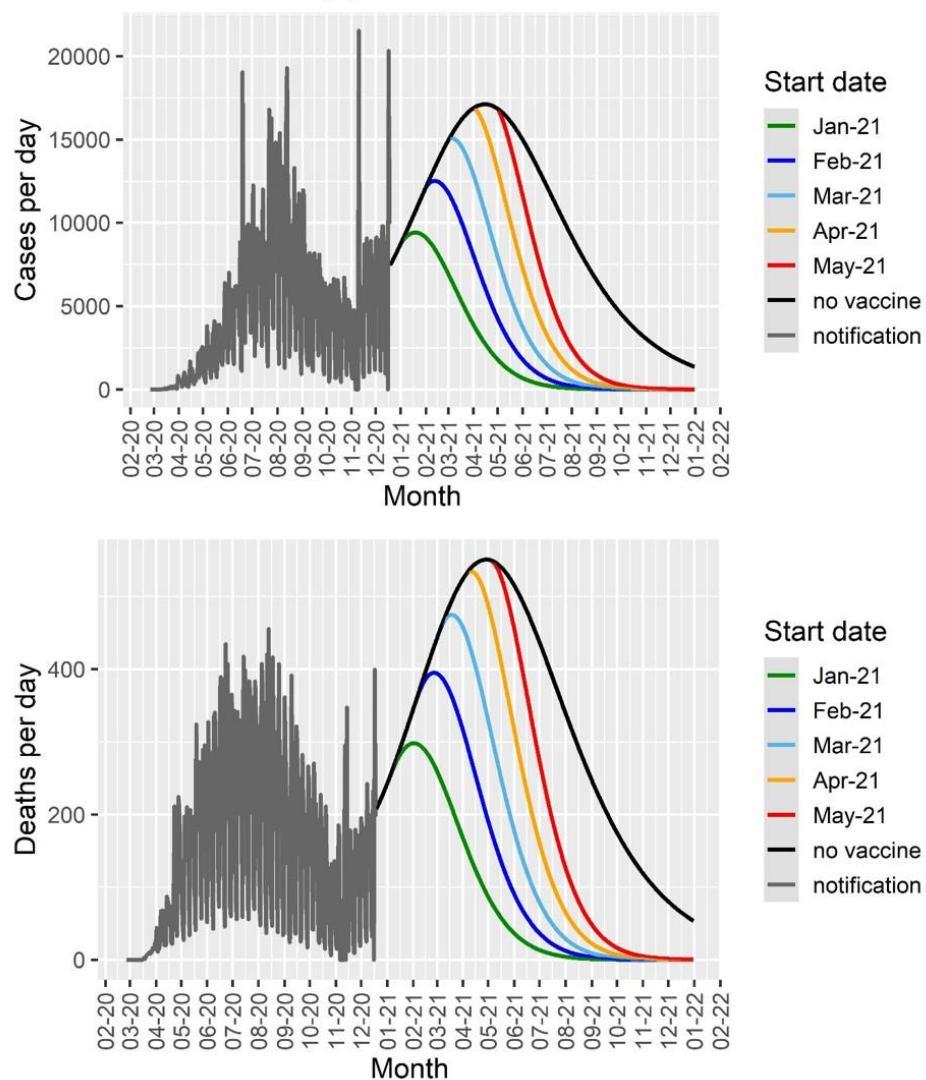

Figure 7 - Cases and deaths per day as a function of time for different start dates for the vaccination campaign in (a) Brazil and (b) the State of Sao Paulofor a vaccination rate of $0.005 \mathrm{day}^{-1}$, and the results for the model with no vaccination (black lines). The notification data until December $18^{\text {th }} \mathbf{2 0 2 0}$ are shown in gray.

In tables 2 and 3 we summarize our main results for one particular scenario maximizing the vaccination rate, compliance of the population (80\%) and vaccine efficacy (90\%), that is, what we should expect in the optimal condition. The simulations are for Sao Paulo and Brazil as a whole.

Table 2. Total number of expected deaths by 31 December 2021 for an optimized vaccination Total deaths until 31 December 2021

\begin{tabular}{|cc|c|}
\hline & São Paulo & Brazil \\
\hline No Vaccination & 168,290 & 352,931 \\
\hline Beginning vaccination on 21 $1^{\text {st }}$ anuary 2021 & 55,489 & 225,289 \\
\hline Beginning vaccination on 21 ${ }^{\text {st }}$ February 2021 & 67,118 & 266,474 \\
\hline Beginning vaccination on 21 $1^{\text {st }}$ March 2021 & 80,384 & 298,341 \\
\hline Beginning vaccination on 21 $1^{\text {st }}$ April 2021 & 97,093 & 322,580 \\
\hline Beginning vaccination on 21 $1^{\text {st }}$ May 2021 & 113,545 & 336,475 \\
\hline
\end{tabular}




\begin{tabular}{|c|c|c|}
\hline & \multicolumn{2}{|c|}{$\begin{array}{l}\text { Expected number of deaths due to vaccination } \\
\text { delay until } 31 \text { December } 2021\end{array}$} \\
\hline & Sao Paulo & Brazil \\
\hline 1 month delay & 11,629 & 41,185 \\
\hline 2 months delay & 24,895 & 73,052 \\
\hline 3 months delay & 41,604 & 97,291 \\
\hline 4 months delay & 58,056 & 111,186 \\
\hline
\end{tabular}

It can be noted from table 2 that, in the absence of vaccination, the model projects almost 170 thousand deaths and more than 350 thousand deaths until the end of 2021 for Sao Paulo and Brazil, respectively. If in contrast, Sao Paulo and Brazil had enough vaccine supply and so started a vaccination campaign in January with the maximum vaccination rate, compliance and efficacy, they could have averted more than 112 thousand deaths and 127 thousand deaths, respectively.

In table 3 we show the number of additional deaths attributable to vaccination delay. It can be seen that for each month of delay the number of deaths increases monotonically (in a logarithm fashion) for both the State of Sao Paulo and Brazil as a whole.

\section{Discussion}

In this paper we present a theoretical exercise, represented by a model intended to estimate the impact of (perhaps inevitable) delay in starting vaccination against SARS-CoV-2, illustrated with the epidemic situation in Brazil and in the State of Sao Paulo. The model parameters are calibrated from reports of daily COVID-19 infections, as well as published reports and it reproduces the real data with remarkable accuracy. Our results demonstrate that, both for Brazil as a whole and for the State of Sao Paulo, for each month of delaying the starting of vaccination, the number of deaths to COVID-19 is staggering high.

We assumed vaccination rates that simulates immunization of up to $70 \%$ of the whole country in 9 months, which may seem unfeasible but Brazil has a long tradition in mass vaccination campaigns [21], managing to immunize more than 20 million people in a single day [22]. Therefore, the maximum vaccination scenario would be a real possibility, given the country experience in mass vaccination schedules adopted in the past. However, due to difficulties in vaccine acquisition, the number of available doses so far has been very low indeed [23]. At the time of writing, Brazil has 
vaccinated slightly above $2 \%$ of its population, way below the target of at least $70 \%$ to achieve the assumed herd immunity level.

The model has some important limitations worth mentioning, the most important is perhaps that it does not consider age-dependence in incidence of the infection and in the mortality rates. However, the model was intended to simulate a mass vaccination campaign that would include all age strata in a relatively short period of time. In addition, we considered only the original variant of the virus, which means that our results represent a lower bound in the number of cases and deaths due to vaccination delay. The current scenario of the pandemic, in which new variants of SARS-CoV-2 are emerging in some countries [24] should be considered in the simulation of future vaccination models, but there is not enough empirical evidence of the impact of these new variants as related to the vaccine efficacy.

Other important limitation is that in the model only having one vaccine dose is considered, however many of the current vaccines require two doses. This assumption that everyone just requires one dose to be fully immunized was done to simplify the calculations, and despite the fact that many vaccines require two doses, this would not significantly change the results of the model. In future models we intend to apply a more realistic scenario with two doses of the vaccine.

Reported cases, deaths and number of ICU beds were fitted simultaneously for Sao Paulo, while only cases and deaths were fitted for Brazil, because a dataset with the daily number of ICU patients was only available for Sao Paulo. As a result, the accuracy of the parameter estimates related to hospitalized patients in Brazil may have been affected.

Finally, we should note that the vaccination rate currently applied in Brazil is estimated to be 10 times less than the one applied, for instance in Israel. With the current vaccination rates it will take more than a year to reach herd immunity in Brazil.

\section{Conclusion}

In conclusion, our model shows that the current delay in the vaccination schedules, that is observed in many countries, has serious consequences in terms of mortality by the disease and should serve as an alert to health authorities to speed the process up such that the highest number of people to be immunized is reached in the shortest period of time. 


\section{List of abbreviations}

COVID-19: Coronavirus induced disease 2019.

SARS-CoV-2: Severe acute respiratory syndrome by the coronavirus 2 .

USA: United States of America.

$S(t)$ : Susceptible individuals.

$V(t)$ : Vaccinated individuals.

$F V(t)$ : Failure to be immunized individuals.

$E(t)$ : Exposed individuals.

$A(t)$ : Asymptomatic individuals.

$I(t)$ : Infective individuals.

$H(t)$ : Hospitalized individuals.

$G(t)$ : Grave individuals.

$R(t)$ : Recovered individuals.

$R_{0}$ : Basic Reproduction Number.

ICU: Intensive Care Unit.

Ethics approval and consent to participate: Not applicable.

Consent to publication: Not applicable.

Availability of data and materials: The dataset used and analysed during the current study are available from the corresponding author on reasonable request.

Competing interest: The authors declare that they have no competing interest.

Funding: This work was partially supported by LIM01-HFMUSP, CNPq, FAPESP and Fundação Butantan.

Authors' contributions: All authors contributed equally for this study. All authors read an approved the final manuscript.

Acknowledgments: The authors thank the staff of LIM01-HCFMUSP and Fundacao Butantan for their infrastructure support. 


\section{References}

1. https://www.who.int/emergencies/diseases/novel-coronavirus-2019/covid-19-vaccines. Accessed 18 February 2021.

2. https://ourworldindata.org/covid-vaccinations. Accessed 18 February 2021.

3. https://www.bloomberg.com/graphics/covid-vaccine-tracker-global-distribution/. Accessed 18 February 2021.

4. https://www.bloomberg.com/graphics/covid-vaccine-tracker-global-distribution/. Accessed 18 February 2021.

5. T. McKeon e R. G. Brown, "Medical evidence related to English population changes in the eighteenth century," Population Studies,vol. 9, pp. 119-141, 1955.

6. T. McKeon e C. R. Lowe, An Introduction to Social Medicine, Oxford and Edinburgh: Blackwell Scientific Publications, 1966.

7. N. A. Christakis, Appolo's Arrow: The Profound and Enduring Impact of Coronavirus on the Way we Live, New York: Little, Brown Spark, 2020.

8. E. S. Pronker, T. C. Weenen, H. Commandeur, E. Cloassen e A. Osterhaus, "Risk in vaccine research and development quantified," PLoS One, vol. 8, p. e57755, 2013.

9. K. Arastu, "A brief introduction to Covid-19 vaccines," January 2021. [Online]. Available: domlipa.ca/sites/default/files/Covid-Vaccines.pdf. [Acesso em 04 January 2021].

10. https://covid19.trackvaccines.org/vaccines. Accessed 18 February 2021.

11. https://www.bloomberg.com/graphics/covid-vaccine-tracker-global-distribution/. Accessed 18 February 2021.

12. James LP, Salomon JA, Buckee CO, Menzies NA (2021). The use and misuse of mathematical modeling for inteftious disease policymaking: lessons for the COVID-a9 pandemic. Medical Decision Making 41(4): 379-385.

13. Bubar KM, Reinholt K, Kissler SM, Lipsitch M, Cobey S, Grad YH, Larremore DB. (2021). Model-informed COVID_19 vaccine prioritization strategies by age and serostatus. Science 371: 916-921.

14. Moore S, Hill EM, Tildesley MJ, Dyson L, Keeling MJ. (2021). Vaccination and nonpharmacological interventions for COVID-19: a mathematical modeling study. Lancet Infectious Diseases. Maarch 18, 2021. https://doi.org/10.1016/S1473-3099(21)00143-2

15. https://covid.saude.gov.br/. Accessed 18 February 2021.

16. https://www.seade.gov.br/coronavirus/\#. Accessed 18 February 2021.

17. https://viz.saude.gov.br/extensions/DEMAS_C19Vacina/DEMAS_C19Vacina.html. Accessed 18 February 2021.

18. Massad E, Amaku M, Wilder- Smith A, Costa dos Santos PC, StruchinerCJ,Coutinho FAB (2020). Two complementary model-based methods for calculating the risk of international spreading of a novel virus from the outbreak epicentre. The case ofCOVID-19. Epidemiology and Infection 148, e109, 1-6. https://doi.org/10.1017/S0950268820001223.

19. Amaku M, Covas DT, Coutinho FAB, AzevedoNeto RS, Struchiner CJ, Wilder-Smith A, Massad E. Modelling the test, trace and quarantine strategy to control the COVID19 epidemic in the state of Sao Paulo, Brazil. InfectiousDiseaseModelling 6 (2021) 4655.https://doi.org/10.1016/j.idm.2020.11.004 
20. Amaku M, Covas DT, Coutinho FAB, AzevedoNeto RS, Massad E. Modelling the impact of contact tracing of symptomatic individuals on the COVID-19 epidemic. Clinics. In Press.

21. Massad E, Burattini MN, Azevedo Neto RS, Yang HM, Coutinho FAB, Zanetta DMT. A model-based design of a vaccination strategy against rubella in a non-immunized community of Sao Paulo State, Brazil. Epidemiology and Infection (1994). 112: 579594.

22. https://setorsaude.com.br/no-brasil-93-das-criancas-se-vacinaram-contra-polio-e-sarampo-deacordo-com-ministerio-da-saude/. Accessed 18 February 2021.

23. CDC - https://www.cdc.gov/coronavirus/2019-ncov/transmission/variant.html. Accessed 18 February 2021.

24. https://www.who.int/news-room/feature-stories/detail/the-race-for-a-covid-19 vaccineexplained. Accessed 18 February 2021. 


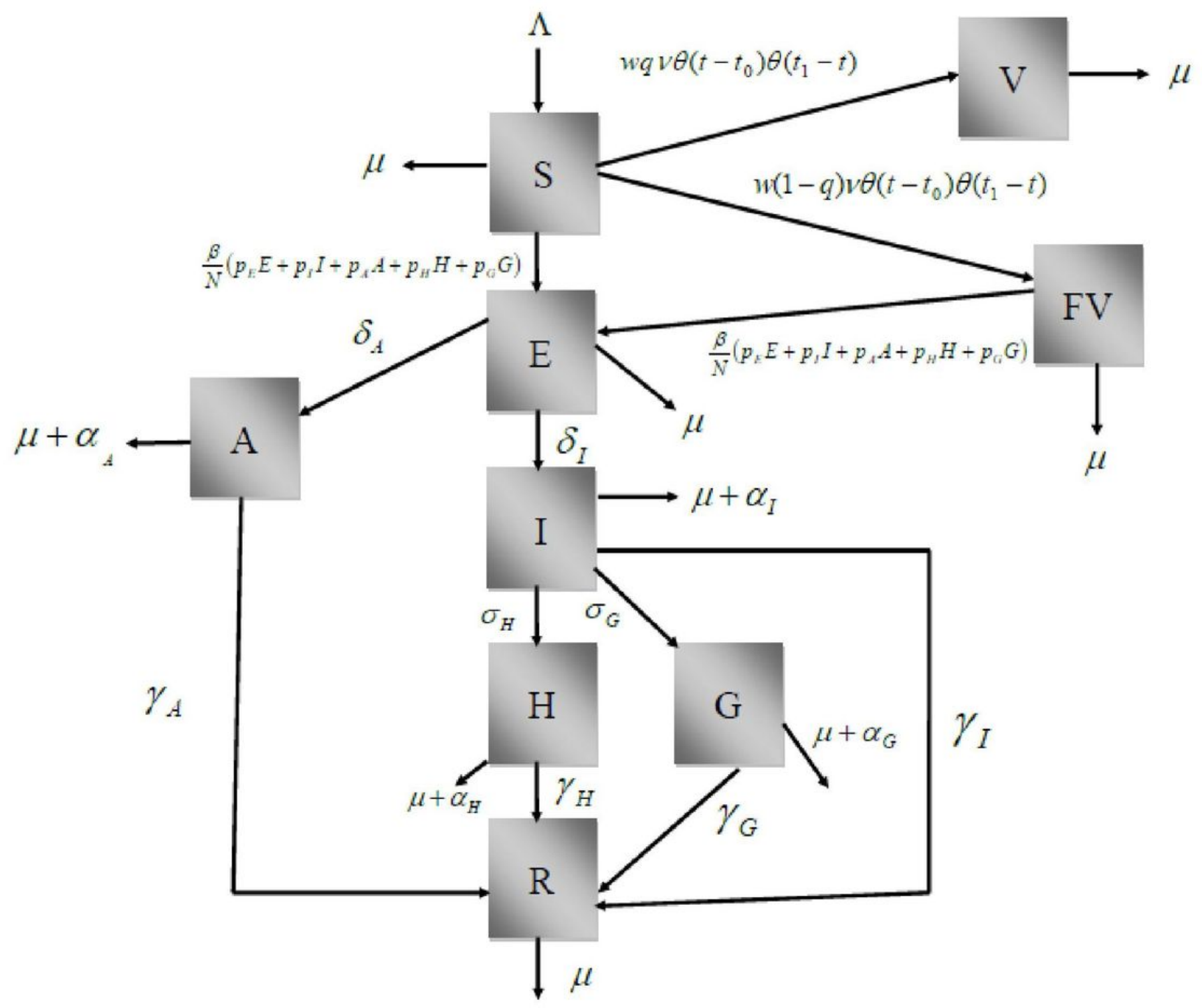

Figure 1

Diagram showing the model's stages and transitions. The figure shows a block diagram with all the model's states, namely, susceptible (S), vaccinated (V), vaccination failure (FV), exposed to the virus (E), asymptomatic (A), infected and infectious (I), hospitalized $(H)$, ICU patients $(G)$ and recovered from infection (R). Transitions from one compartment to other are represented as rates, as described in the main text. 


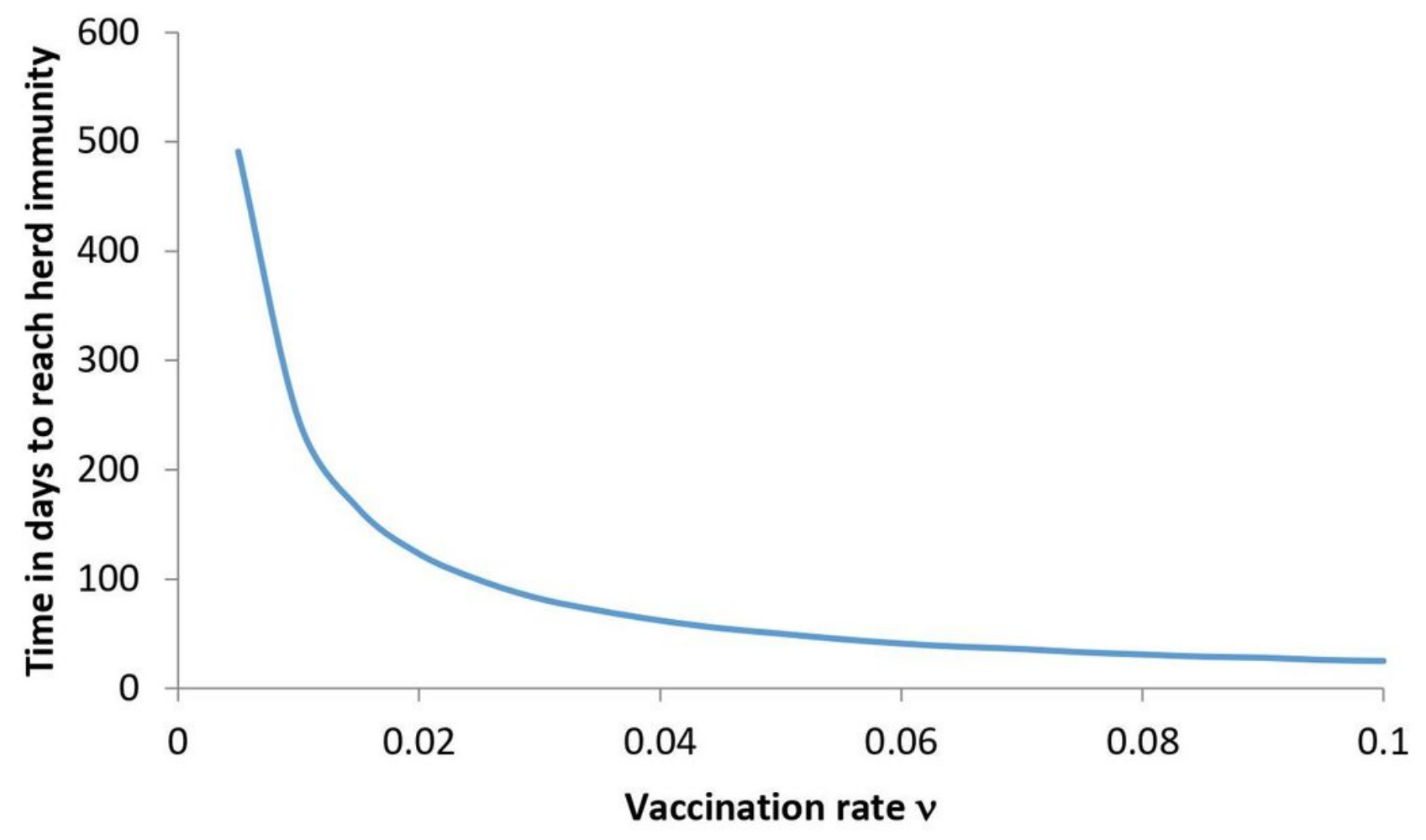

Figure 2

Time in days taken to reach herd immunity as a function of vaccination rates as used in the simulation of the model. 
(a) Brazil
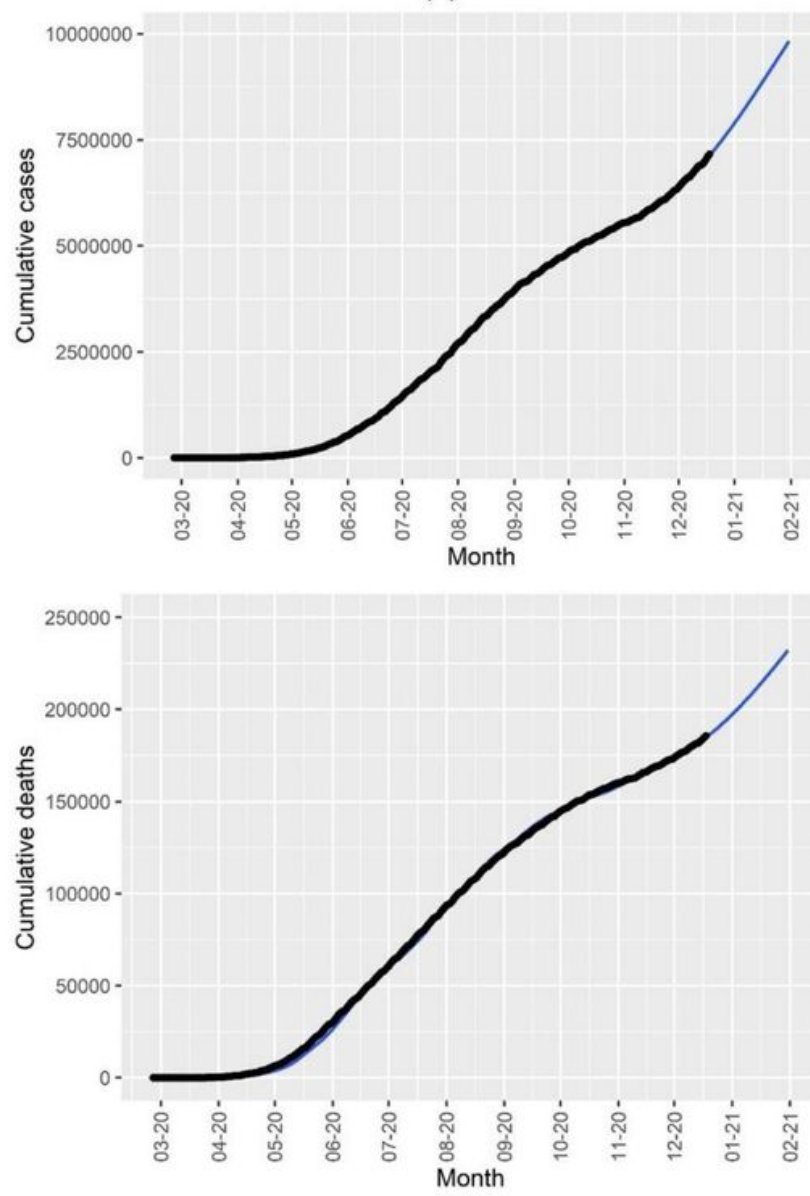

(b) Sao Paulo
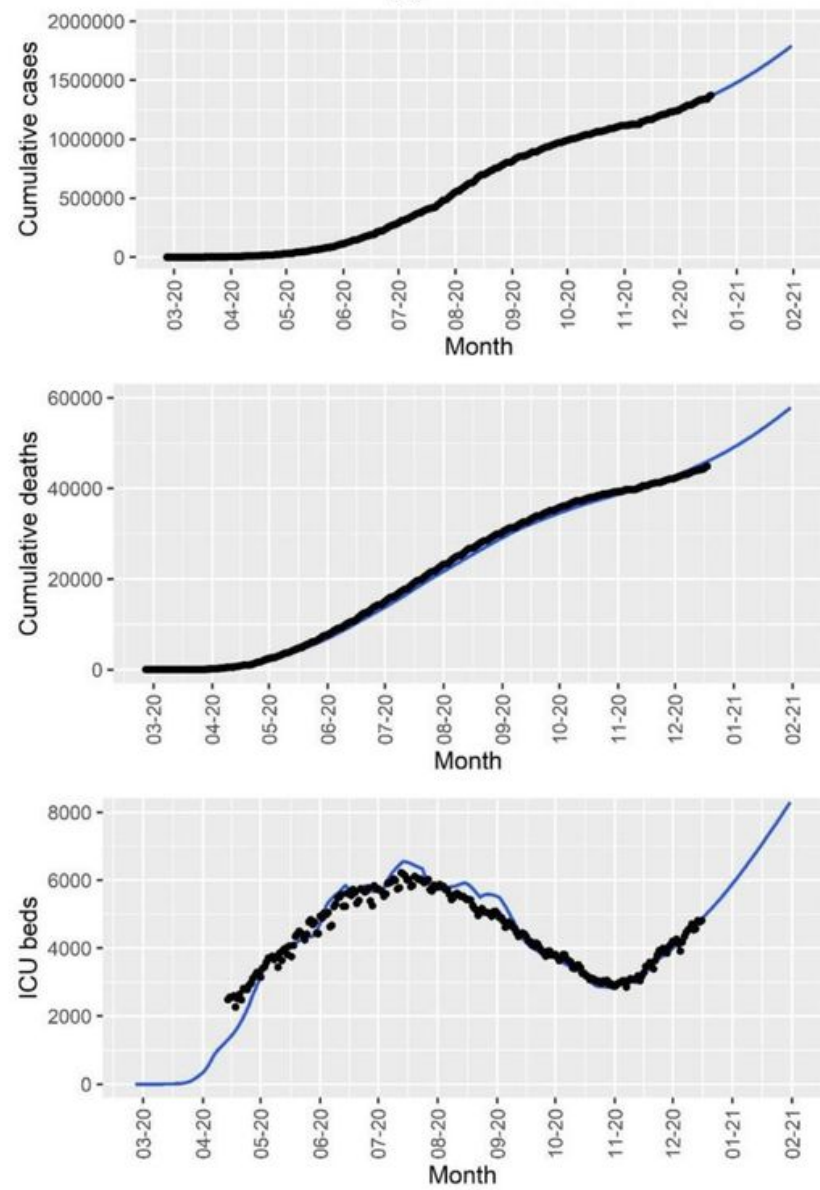

\section{Figure 3}

Cumulative number of reported cases and deaths in (a) Brazil (black dots) and cases, deaths, and number of ICU patients in (b) the State of Sao Paulo. The blue lines correspond to the fitted models. 
(a) Brazil

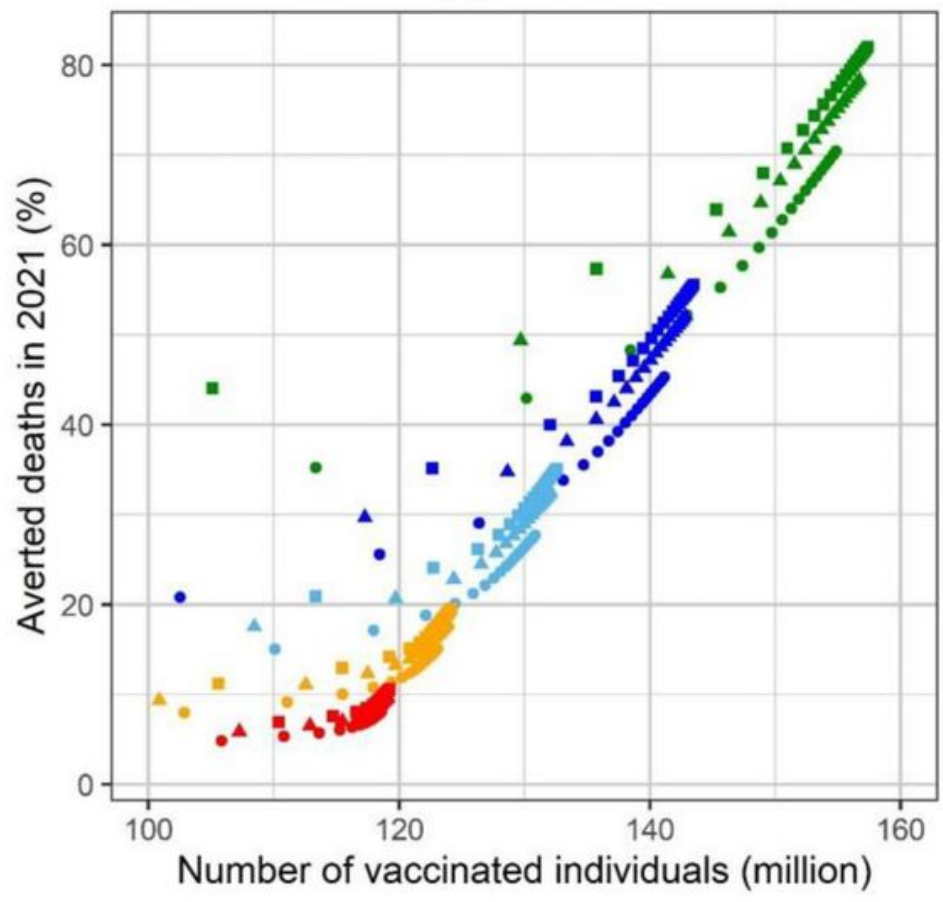

(b) Sao Paulo

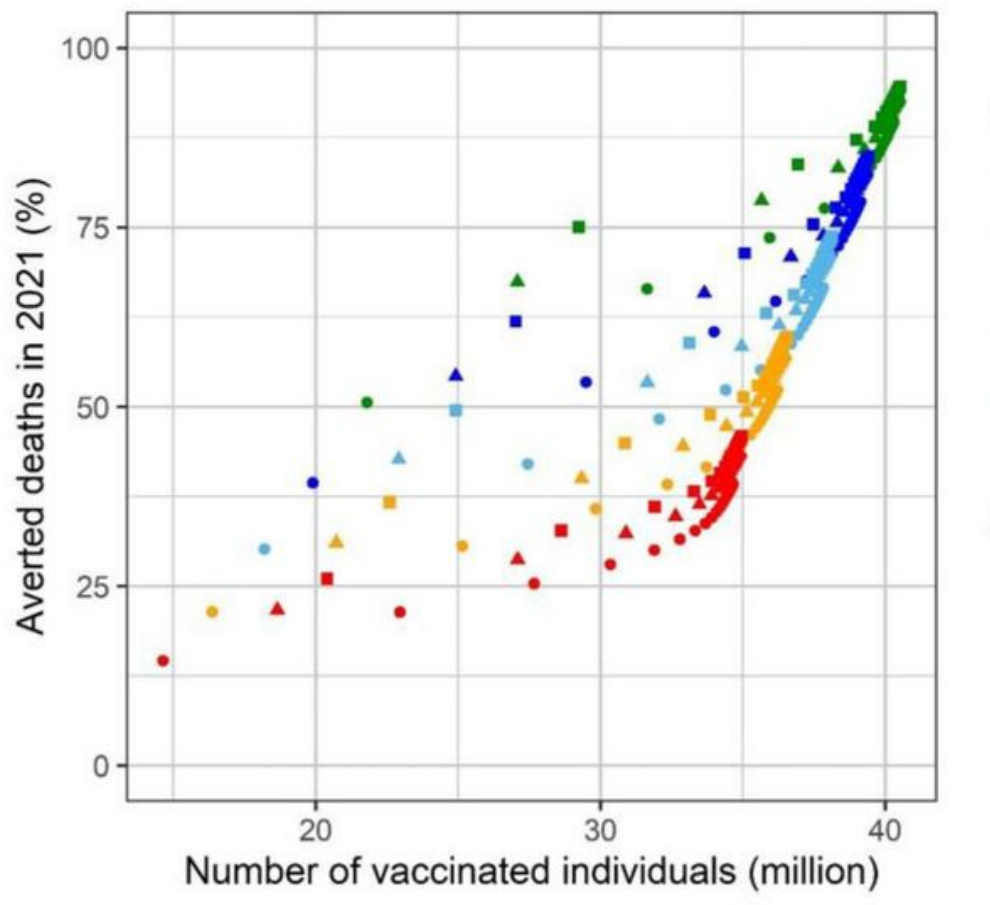

Start date

- Jan-21

- Feb-21

- Mar-21

- Apr-21

- May-21

Adherence (w) efficacy (q)

- $w=0.5 q=0.5$

- $w=0.7 q=0.7$

- $w=0.8 q=0.9$

\section{Start date}

- Jan-21

- Feb-21

- Mar-21

- Apr-21

- May-21

Adherence (w) efficacy (q)
- $w=0.5 q=0.5$
- $w=0.7 q=0.7$
- $w=0.8 q=0.9$

\section{Figure 4}

Percentage of averted deaths in 2021 in (a) Brazil and (b) the State of Sao Paulo as a function of the number of vaccinated individuals for different start dates for the vaccination campaign. Three different combinations of vaccination adherence $(w)$ and vaccine efficacy $(q)$ were considered: $w=0.8 a n d q=0.9$ (best-case scenario), $w=0.7$ and $q=0.7$ (baseline scenario) and $w=0.5$ and $q=0.5$ (worst-case scenario). 
(a) Brazil

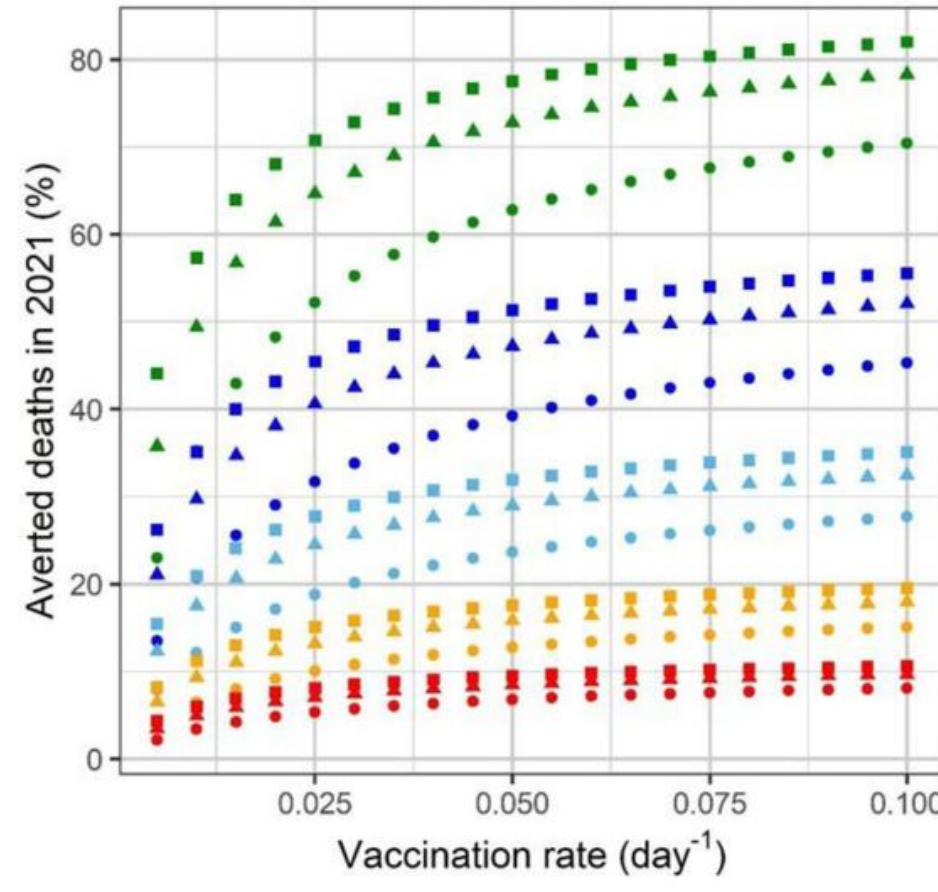

(b) Sao Paulo

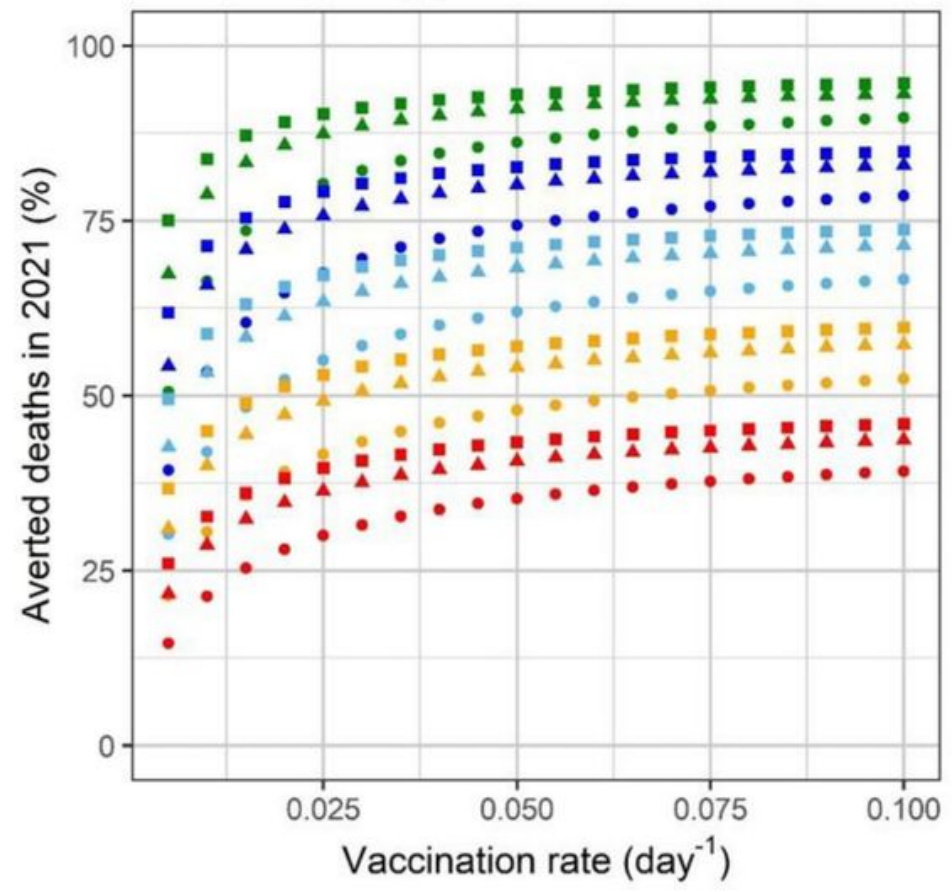

\section{Start date}

- Jan-21

- Feb-21

- Mar-21

- Apr-21

- May-21

Adherence (w) efficacy (q)

- $w=0.5 q=0.5$

- $w=0.7 q=0.7$

- $w=0.8 q=0.9$

\section{Start date}

- Jan-21

- Feb-21

- Mar-21

- Apr-21

- May-21

Adherence (w) efficacy (q)

- $w=0.5 q=0.5$

- $w=0.7 q=0.7$

- $w=0.8 q=0.9$

\section{Figure 5}

Percentage of averted deaths in 2021 in (a) Brazil and (b) the State of Sao Paulo as a function of the vaccination rate for different start dates for the vaccination campaign. Three different combinations of vaccination adherence $(w)$ and vaccine efficacy $(q)$ were considered: $w=0.8$ and $q=0.9$ (best-case scenario), $w=0.7$ and $q=0.7$ (baseline scenario) and $w=0.5$ and $q=0.5$ (worst-case scenario). 
(a) Brazil
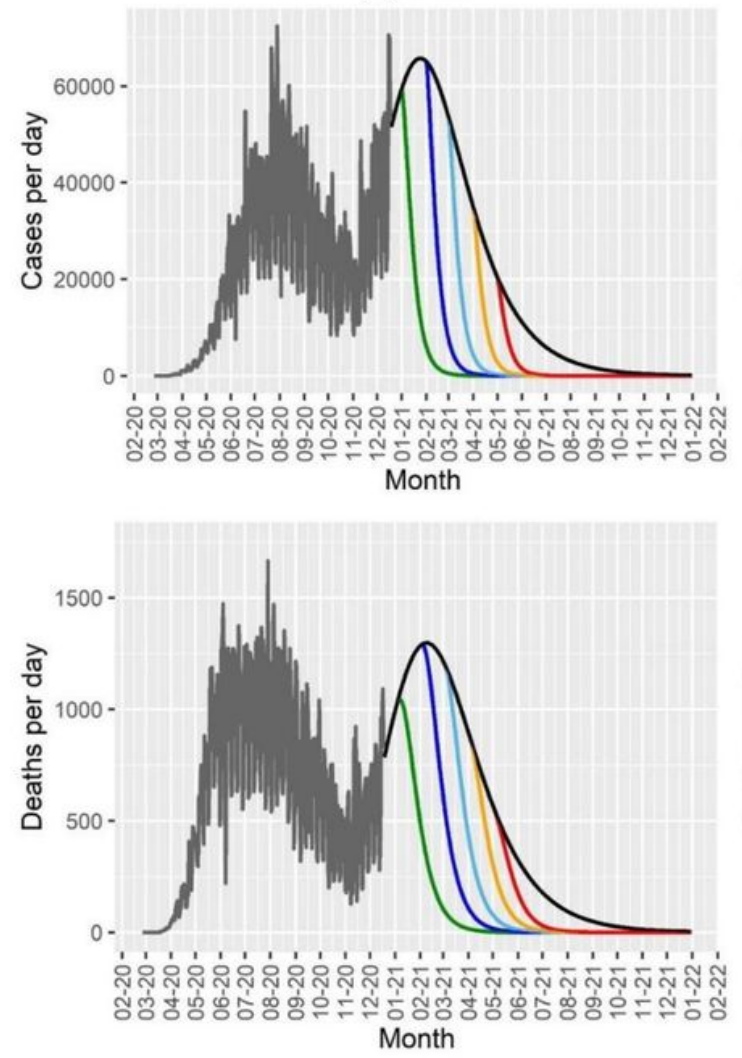

(b) Sao Paulo
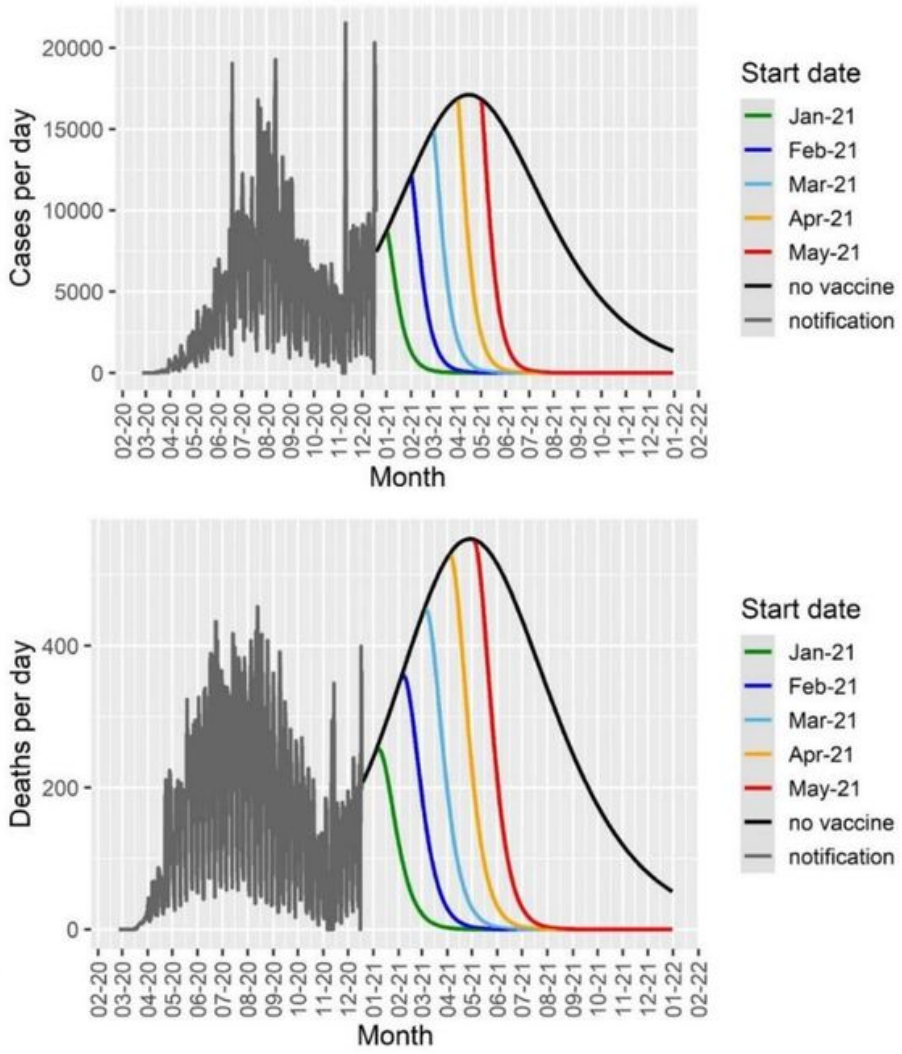

\section{Figure 6}

Cases and deaths per day (b) as a function of time for different start dates for the vaccination campaign in (a) Brazil and (b) the State of Sao Paulo for a vaccination rate of 0.05 day-1, and the results for the model with no vaccination (black lines). The notification data until December 18th 2020 are shown in gray. 
(a) Brazil
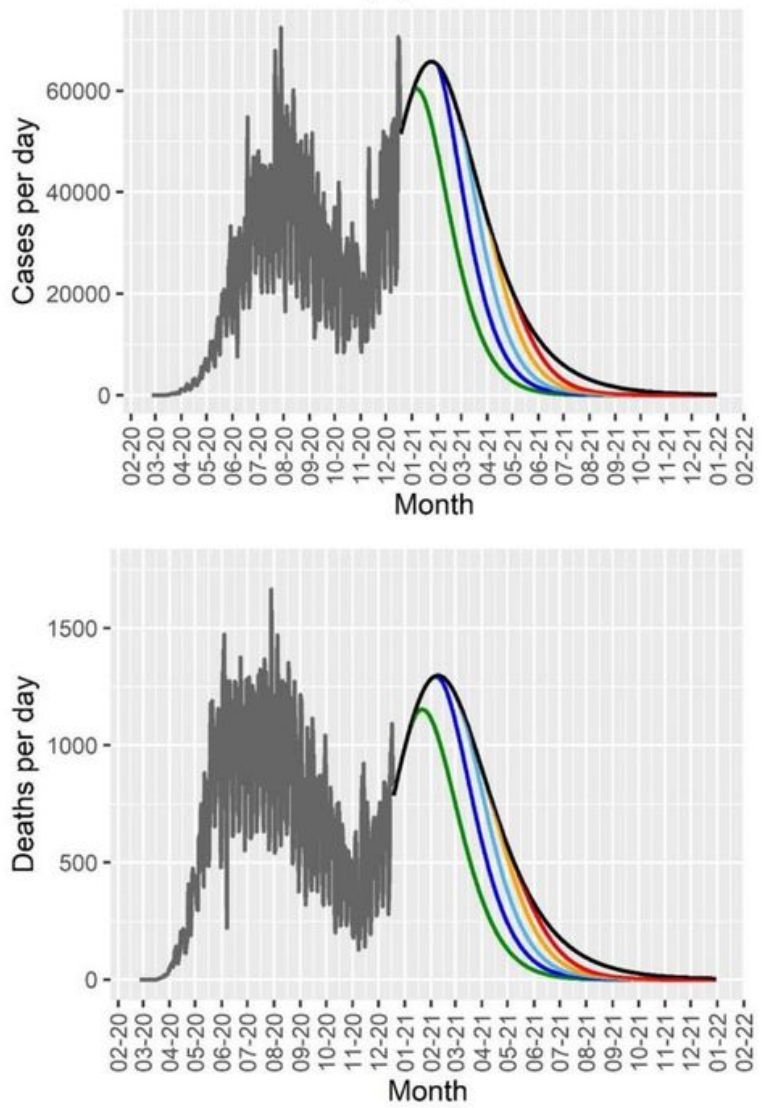

(b) Sao Paulo
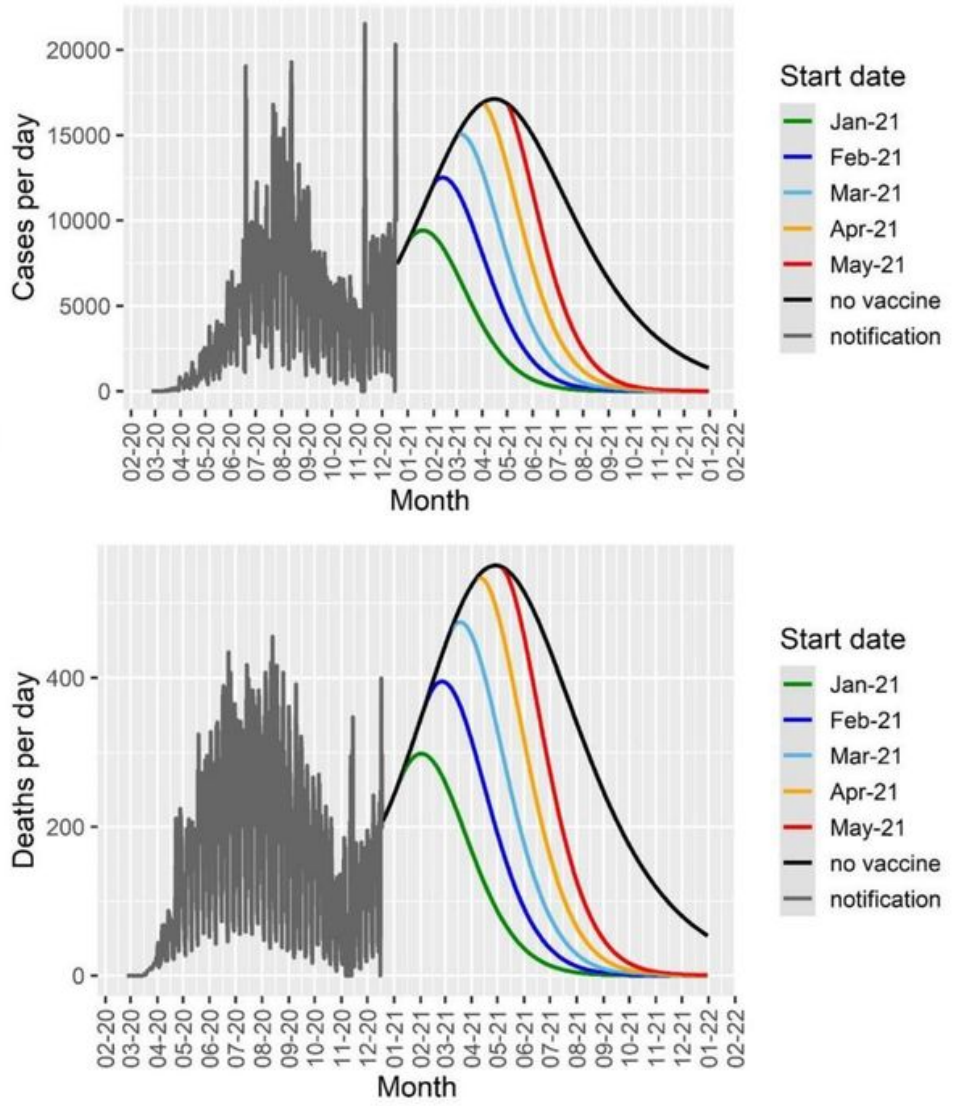

Figure 7

Cases and deaths per day as a function of time for different start dates for the vaccination campaign in (a) Brazil and (b) the State of Sao Paulofor a vaccination rate of 0.005 day-1, and the results for the model with no vaccination (black lines). The notification data until December 18th 2020 are shown in gray.

\section{Supplementary Files}

This is a list of supplementary files associated with this preprint. Click to download.

- AnswerstoReviewers.docx 